\title{
Symbiotic human-robot collaborative assembly
}

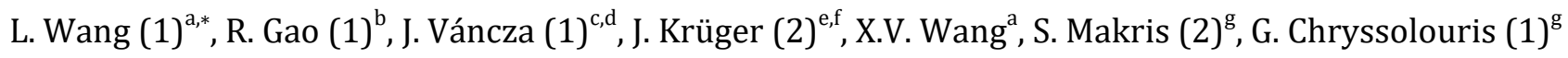 \\ ${ }^{a}$ Department of Production Engineering, KTH Royal Institute of Technology, Stockholm, Sweden \\ ${ }^{b}$ Department of Mechanical and Aerospace Engineering, Case Western Reserve University, Cleveland, OH, USA \\ ${ }^{c}$ Institute for Computer Science and Control, Hungarian Academy of Sciences, Hungary \\ ${ }^{d}$ Department of Manufacturing Science and Engineering, Budapest University of Technology and Economics, Budapest, Hungary \\ ${ }^{e}$ Institute for Machine Tools and Factory Management, Technische Universität, Berlin, Germany \\ ${ }^{f}$ Fraunhofer Institute for Production Systems and Design Technology, Berlin, Germany \\ ${ }^{g}$ Laboratory for Manufacturing Systems \& Automation, University of Patras, Patras, Greece
}

\begin{abstract}
In human-robot collaborative assembly, robots are often required to dynamically change their pre-planned tasks to collaborate with human operators in a shared workspace. However, the robots used today are controlled by pre-generated rigid codes that cannot support effective human-robot collaboration. In response to this need, multi-modal yet symbiotic communication and control methods have been a focus in recent years. These methods include voice processing, gesture recognition, haptic interaction, and brainwave perception. Deep learning is used for classification, recognition and context awareness identification. Within this context, this keynote provides an overview of symbiotic human-robot collaborative assembly and highlights future research directions.
\end{abstract}

Assembly, Robot, Human-robot collaboration

\section{Introduction}

Human-robot collaboration (HRC) in a manufacturing context aims to realise an environment where humans can work side by side with robots in close proximity. In such a collaborative setup, the humans and the robots share the same workspace, the same resources, and in some cases the same tasks. The main objective of the collaboration is to integrate the best of two worlds: strength, endurance, repeatability and accuracy of the robots with the intuition, flexibility and versatile problem solving and sensory skills of the humans. Using HRC, higher overall productivity and better product quality can be achieved. In any HRC system, human safety is of paramount importance.

In the last decade, research efforts on HRC have been numerous. Varying approaches to facilitating multimodal communication, dynamic assembly planning and task assignment, adaptive robot control, and in-situ support to operators have been reported in the literature. Nevertheless, confusions exist in the relationships between robots and humans: coexistence, interaction, cooperation, and collaboration. The roles of humans when working with robots are less clear. The lack of standards and safety solutions results in a low acceptance of the human-robot combination. A systematic review and analysis on this very subject is needed, which is the motivation and objective of this keynote paper.

This paper starts with a classification of the human-robot relationships and then provides detailed treatments on relevant issues with a focus on symbiotic HRC assembly. The remainder of this paper is organised as follows: Section 2 gives the definition and characteristics of HRC after classifying the human-robot relationships; Section 3 reviews the existing technologies for sensing and communication in HRC; Section 4 introduces the available safety standards and systems for collision avoidance; Section 5 presents reported solutions for dynamic context-aware task planning and assignment, assisted by deep learning; Section 6 provides insights on programming-free adaptive robot control through algorithm embedding and a brainwave-driven method;
Section 7 reveals different techniques and systems for mobile worker assistance. Section 8 points out the remaining challenges and future research directions; and finally, Section 9 concludes this keynote paper. Depending on the context, worker, operator and user are used to represent a human working with a robot.

\section{Classification, definition and characteristics}

\subsection{Classification of human-robot relationships}

The relationship of humans and robots in a shared work environment is a many-faceted phenomenon which is classified according to a number of different viewpoints [96]. Schmidtler et al. [220] analysed a human-robot cell in terms of working time, workspace, aim and contact. Wang et al. [257] identified workspace, direct contact, working task, simultaneous process, and sequential process as the shared contents between a robot and a human. In general, shared workspace refers to whether the human and the robot are working in the same working area with no physical or virtual fences for separation. Direct contact indicates whether there is a direct physical contact between the robot and the human. Shared working task represents whether the human and the robot work on the same operation towards the same working objective. Simultaneous process means that the human and the robot are working at the same time, but the task can be the same or different. In contrast, the sequential process indicates that the operations of the human and the robot are arranged one after another with no overlap in the temporal scale. Accordingly, the classification of human-robot relationships can be summarised as follows (also in Table 1).

- The basic situation is coexistence when a robot and a human are placed within the physical space but without overlapping each other's workspace. There is no direct contact between the human and the robot. The work object might be exchanged between them, but the process is performed independently and simultaneously. 
- Interaction happens if a human and a robot sharing the same workspace are communicating with each other. One party guides or controls the other, or any physical contact (either planned or unintended) occurs between them. Both the human and the robot can work on the same task but complete the task step by step in a sequential order.

- Cooperation can be developed among human and robot agents who have their own autonomy (expressed in terms of goals, objectives, utility or profit) [237]. In the hope of mutual benefit, cooperating agents may temporarily share some of their physical, cognitive or computational resources, even though they are pursuing their own interests. The parties can share a partially overlapping workspace but direct contact is not typical between them. They can work simultaneously, but at times have to wait for the availability of the other agent(s).

- Collaboration is the joint activity of humans and robots in a shared workspace, with the definite objective to accomplish together a set of given working tasks. It requires typically a coordinated, synchronous activity from all parties [177] where physical contact is also allowed. In any case, collaboration assumes a joint, focused goal-oriented activity from the parties who share their different capabilities, competences and resources.

Table 1. Features of different human-robot relationships.

\begin{tabular}{|c|c|c|c|c|c|}
\hline & & Coexistence & Interaction & Cooperation & Collaboration \\
\hline \multirow{6}{*}{ 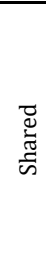 } & Workspace & & $\checkmark$ & $\checkmark$ & $\checkmark$ \\
\hline & Direct contact & & $\checkmark$ & & $\checkmark$ \\
\hline & Working task & & $\checkmark$ & & $\checkmark$ \\
\hline & Resource & & & $\checkmark$ & $\checkmark$ \\
\hline & $\begin{array}{l}\text { Simultaneous } \\
\text { process }\end{array}$ & $\checkmark$ & & $\checkmark$ & $\checkmark$ \\
\hline & $\begin{array}{l}\text { Sequential } \\
\text { process }\end{array}$ & & $\checkmark$ & $\checkmark$ & \\
\hline
\end{tabular}

\subsection{Definition of human-robot collaboration}

In the context of production and according to the standard terminology, HRC is a 'state in which a purposely designed robot system and an operator work on simultaneous tasks within a collaborative workspace', i.e., where the robot system and a human can perform tasks concurrently or even jointly [84]. This implies that there is no temporal or spatial separation of the robotic and humans' activities (like semaphores or fences).

HRC is motivated by a number of factors: the combination of complementary human and robotic skills and intelligence holds the promise of increased productivity, flexibility and adaptability, increased robustness and higher degree of resilience, improved ergonomics, and more attractive work conditions. HRC is also in demand in distributed manufacturing work environments and systems due to the limitation of automation and the maturation of agent technologies [170]. While it is broadly assumed that robotic agents may be multiple and form a team, at present HRC typically only involves a single human - the operator or worker.

HRC has been a subject of systematic analysis and classification efforts both in the general sense [261] and also with a special concern on production and assembly [256]. Due to its specific constraints, industrial production usually occupies a subset of possibilities. Key properties that define distinct classes of HRC instances across all applications are multiplicity and autonomy. Agent multiplicity distinguishes single, multiple, and team settings (Fig. 1), the latter being a group acting together by consensus or coordination, and interacting with the environment and other agents in a specified way (e.g., via a "speaker"). Multiple agents can compete for tasks, resources and/or other agents' services (e.g., one robot serving several manned workstations).

Agent autonomy and closely related leader-follower relationships express how much of robot action is directly determined by human agents, and vice versa. In any case, an agent needs to take the responsibility and leadership when performing the given task. Task execution scenarios can be partitioned along the autonomy of participating agents (see Fig. 2). During task execution, either the human or the robot may assume an active (leading) role, or only support it (as a follower, performing auxiliary actions on-demand, serving as a fixture, etc.) or behave inactively (not taking part in the task, merely being present as an obstacle). Adaptive robots and intuitive humans are able to reassign leader/follower roles on-the-fly. With some few exceptions $[24,177]$, recent research assumes that the roles are assigned before task execution.

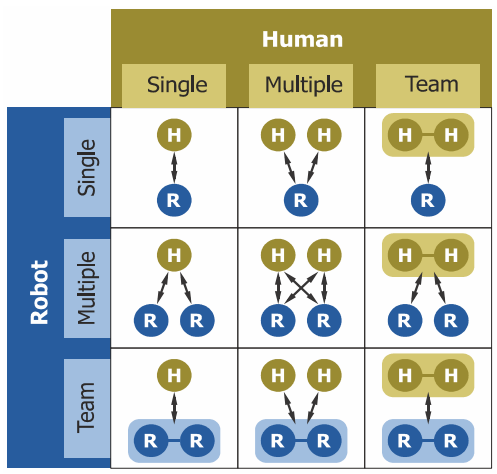

Fig. 1. Possible cases of the human and robotic agents' multiplicity [256].

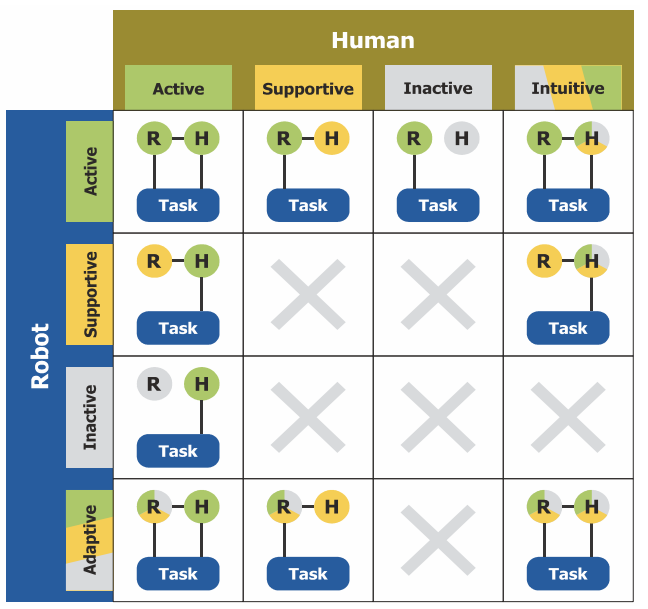

Fig. 2. Possible combinations of the human and robotic agents' roles (adapted from [256]).

\subsection{Symbiotic human-robot collaboration}

Symbiotic cognitive computing takes place when human and machine agents co-exist in a physical space to interact with each other so as to solve hard tasks requiring large amounts of data along with significant mental and computational effort. Such tasks are typically information and knowledge discovery, situation assessment, and strategic decision making [57,93,125]. A modern symbiotic cognitive environment is equipped with a number of multimodal communication techniques such as displays, tablets and cameras, microphones and speakers, motion and haptic sensors, speech and gesture recognition devices that facilitate the context-dependent presentation, manipulation, and analysis of data. The main emphasis is on directly interacting with data as easily, directly, and naturally as possible. The original idea goes back to 1960 when a tentative forecast was made as of the role of computers in supporting complex human decision-making processes. In such a setting, problems requiring intuition from the human's side could be solved better or more effectively would computers be interactive, cooperative and able to turn up flaws of reasoning or reveal unexpected turns in the course of the solution process [126]. By now, state-of-the-art sensor and communication technology, accompanied with almost unlimited data storage capacities and computing power serving faculties of data analytics and reasoning made this vision a reality [57]. 
Symbiotic human-robot collaboration places the interplay of human and machine into a cyber-physical environment [171,182] where human and robotic agents interact in a shared work environment to solve some complex tasks which require the combination of their best, complementing competencies. They form a society of agents which is capable of solving problems the individual members alone would not be able to tackle in a dynamic, as well as only partially structured, observable and predictable work environment. The main traits of symbiotic HRC are the following (see also [57]). (1) While all the parties possess their own autonomies, they form together a team or group which is responsible for the successful and efficient performance of a set of tasks. Leadership, and in general, roles are assumed and changed dynamically, as the actual situation and the tasks require (see Fig. 2, bottom right cell). (2) The agents are context-aware, i.e., their actions and decisions are grounded on the actual physical and cognitive circumstances. The shared work environment provides the ways for all parties to communicate their availability, and offers means for identifying humans, following their activity and tracking and tracing the objects - both physical and computational - they manipulate. (3) The symbiosis continuously engages humans and robots with each other, in an ongoing manner. Multimodal and bidirectional communication is supported between any two or multiple parties, by removing cognitive barriers, distractions, and interruptions as far as possible. (4) The agents apply at least partially shared representations of the environment they are operating in, which is the prerequisite for aligning their (joint) goals, roles, plans and activities. This shared virtual representation should be mapped via sensors dynamically, in real-time with the physical production environment and its temporal evolution, providing its digital twin. (5) The performance of a symbiotic system improves over time. Hence, the environment provides performance feedback to all parties who have a capability to adapt to changing conditions and to learn both from failures and successes. (6) Finally, in the shared work environment, safety of human agents is warranted even under unexpected conditions.

All in all, a symbiotic HRC system possesses the skills and ability of perception, processing, reasoning, decision making, adaptive execution, mutual support and self-learning through real-time multimodal communication for context-aware human-robot collaboration. Compared with fully automated systems and purely manual operations, symbiotic HRC combines the skills of humans and robots, and offers the opportunity of improved manufacturing performance. Better work ergonomics can also be achieved with the help of flexible in-situ operator supports.

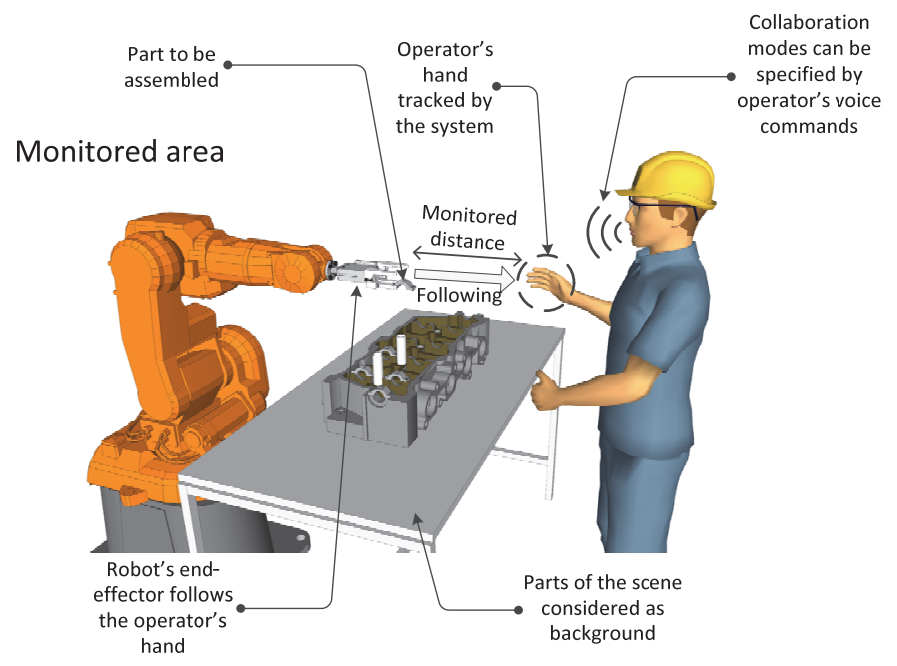

Fig. 3. Multimodal symbiotic human-robot collaboration [166].

However, today's robot control approach based on rigid native codes can no longer support symbiotic HRC. The smartness of a symbiotic HRC system must be enabled by a new means that is multimodal and symbiotic to facilitate any changes during collaboration. Fig. 3 illustrates an exemplified symbiotic HRC system that is driven by voice commands, gesture instructions, haptic interactions, and even human thoughts captured in the form of brainwaves, in a shared setting.

\subsection{Characteristics of HRC assembly}

During HRC assembly, objects are arranged in space by actions in time so that products specified by design can be realised. The space is densely populated not only by parts of the product but also by the applied technological resources and humans, whereas key objectives require the execution of actions within as short a timeframe as possible. Objects and actions involved in HRC assembly are strongly related and constrain each other in many ways, due to technology, product structure, and geometry $[100,101]$. In assembly, the workplace design that allows efficient and dynamic human-robot task allocation is characteristic to safe, ergonomic and symbiotic HRC assembly [163].

Given an assembly environment, task allocation to robots and humans may change over time due to availability and suitability of the resources against the allocated time. This characteristic of human-robot task assignment was investigated and modelled as a search problem [233]. An intelligent decision making method was implemented using a tree representation to derive efficient task assignments between humans and robots, enabling the allocation of sequential tasks assigned to a robot and a human in separate workspaces (Fig. 4). The focus is rather given to the human-robot coexistence for the execution of sequential tasks, in order for the automation level in manual or even hybrid assembly lines to be increased.

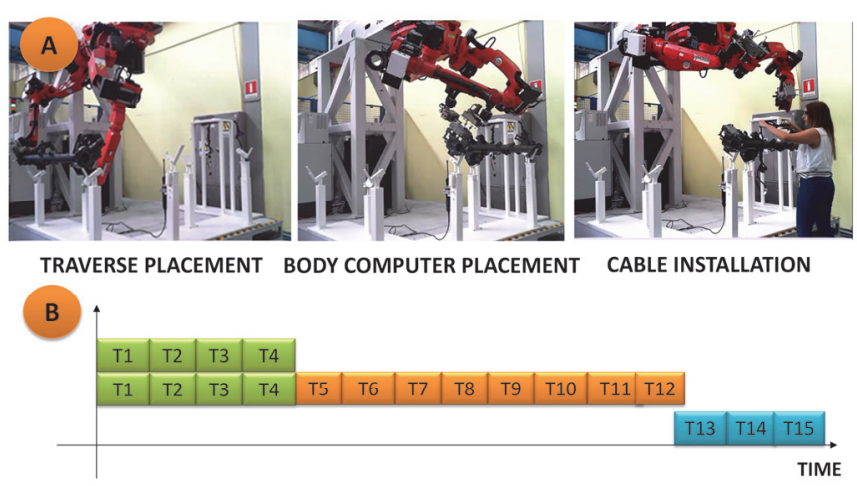

Fig. 4. A) Dashboard assembly case, B) Gantt chart of the best alternative [233].

\section{Sensing and communication}

\subsection{Sensor}

In a collaborative environment where human and robot occupy the same workspace at the same time, a robot requires accurate information regarding human intention, physical parameters at points of haptic interaction, as well as geometric interpretation of the environment to (1) carry out effective HRC tasks, and (2) to comply with the safety aspects for collaborative operation outlined by International Organisation for Standardisation (ISO): safety-rated monitored stop, hand-guiding operation, speed \& separation monitoring and power \& force limiting [85]. The need for improving the effectiveness and efficiency as well as reducing the safety risks in HRC has led to increased interest in sensorrelated research and development for HRC.

Sensors deployed in the HRC environment can be categorised into two families: contact-based and contact-less.

\subsubsection{Contact-based sensing}

The main application of physical contact-based sensing is one type of human gesture recognition (using wearable sensors, e.g., 
gloves) as opposed to camera-based gesture recognition methods. Gestures have been an integral part of human communication throughout history. Naturally, the gesture of human hand and its coordination have been at the forefront of research in HRC, resulting in the category of wearable sensors for gesture recognition, serving as an important human-robot interface $[10,36,50,79,191,136]$. A combination of accelerometer and gyroscope has been described by Asokan et al. [10] to sense the orientation of the hand by placing the sensors on the back of the palm, and potentiometer mounted on the acrylic strip attached to the finger to measure the angle as finger moves. However, one of the main issues with traditional wearable sensors is sensor rigidity, which causes issues such as poor adaptability to the hand as well as reduced hand mobility. Thin, elastic materials, on the other hand, can undergo a wide range of reversible deformation and therefore have become the leading candidate in fabricating wearable sensors that are both reliable and comfortable.

For example, Cha et al. [36] integrated flexible polyvinylidene fluoride-based (PVDF) piezoelectric sensors into a glove to detect the angles of finger joints by converting the angular velocity of the finger motions into voltage. The sensor material is lightweight and self-powered, enabled by the piezoelectric effect. The PVDF piezoelectric sensors are further investigated for wrist mount, generating robot control signal through the coordination of hand gestures [50]. The PVDF sensor can be easily delaminated from the skin and reused from one wrist to the other.

Graphene-based piezoelectric sensors [79,191] have also been investigated. These sensors are made of ultrathin nanomaterials and piezoelectric polymers with superior adaptability to the skin and improved aesthetics due to its near transparent appearance. Further advantages include high signal-to-noise ratio and low power consumption. The sensors proposed by Hong et al. [79] have demonstrated the capability of accurately recognising wrist movement such as stretching and compressing. Park et al. [191] further integrated the sensors into an elbow band to detect the elbow bending angle. Similarly, a graphene hybrid structure with ultrafast dynamic pressure response was reported by Liu et al. [136]. The graphene-based resistive pressure sensor is shown to be capable of frequency-independent sensing with no phase-lag. It also has high sensitivity to subtle pressure or movement.
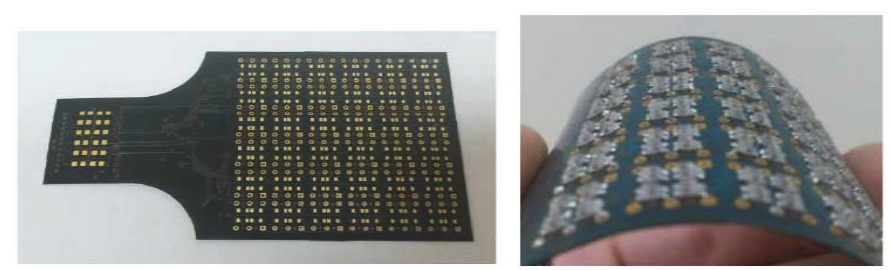

Fig. 5. Pictures of the realised flexible PCB before (left) and after (right) the optoelectronic components mounting [43].

In HRC, robots are expected to physically interact with humans and objects. Being able to sense pressure/force, hardness and texture at the points of contact are essential for both effective/delicate robot reaction when normal contact occurs and compliance to safety requirements during incidental contact. Common scenarios include controlling robot pressure/force such that the object being handled is not damaged and the human is not harmed during incidental contact. The data from the tactile sensor provides the basis for a robot to intelligently reason about the haptic interaction scenario and react appropriately during the contact. Cirillo et al. [43] presented a flexible tactile sensor based on optoelectronic technology to detect both the position of the contact point and the three components of the applied force. The working principle is based on the array of sensing modules, each comprised of four taxels and one optical LED/phototransistor pair, as show in Fig. 5. The deformation of the sensing module under contact pressure produces variations of LED light reflected by the taxels and consequently, the photocurrents that can be measured and used to determine the mechanical stimuli. Li et al. [124] developed a flexible thin tactile sensor based on the dualmode Triboelectric nano-generators (TENGs). Unlike the sensor by Cirillo et al. [43], this sensor is self-powered and can not only detect tiny pressure/force but also distinguish the hardness of the contact material by quantifying the shape change at the current peak. For example, as shown in Fig. 6, in the case of stiff materials such as copper and glass, the current increases suddenly, which is significantly different from the slow and continuous change in the case of soft materials, such as terylene and polydimethylsiloxane (PDMS). A detailed review of various tactile sensors for manipulation and grasping applications was provided by Kappassov et al. [97].

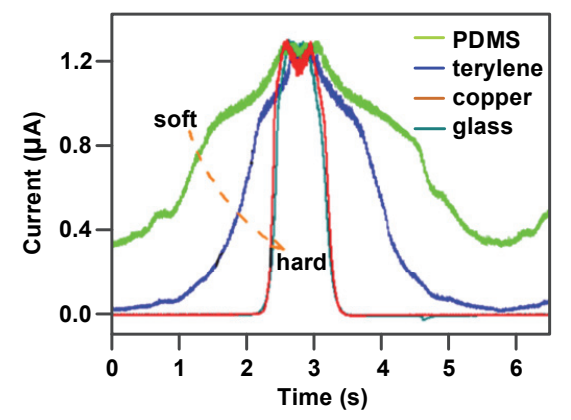

Fig. 6. Shapes of current peak for different contact materials by tactile sensor based on dual-mode Triboelectric nano-generators (TENGs) [124].

\subsubsection{Contact-less sensing}

Contact-less sensing, such as a laser, radar or vision system, helps reconstruct the geometric information of the surroundings in an HRC environment, guiding the robots to move around the workspace avoiding obstacles and work collaboratively with humans by identifying and locating the working parts. In recent years, development of computer vision techniques has enabled context-aware interpretation of the environment, allowing robots to acquire complex skills.

The principles of contact-less sensing can be classified into passive and active methods. In passive sensing, the measurement system does not illuminate the target; instead, the light from the target is either reflected ambient light or the light produced by the target itself. Common passive techniques include traditional optical/infrared camera and stereo vision. Stereo vision is the extension to 3D information from traditional 2D images. It requires the reference point (marker) on the target to be captured by multiple cameras from different projection angles and fused to reconstruct in the 3D space through suitable transformation. In active sensing, the measurement system illuminates the target and captures the pattern of the reflected light. Common active sensing techniques include structured light, time of flight (ToF) and triangulation, which can natively capture the depth information that has to be inferred in passive techniques [197]. In structured light, different light patterns are projected onto the object and a camera captures the reflected patterns from a different angle. By analysing the distortion or curvature information from the pattern, the 3D geometry of the object can be reconstructed. The depth determination from a ToF image is based on the principle of the speed of light. By computing the travel time or phase delay of the reflected light (i.e., a laser pulse), the depth information of each point on the object can be obtained and added to the traditional 2D image. In triangulation, a light beam is first projected on to the surface of the object and a charge-coupled device (CCD) at a different angle receives the reflected light, as illustrated in Fig. 7. As the surface of the object moves away from its initial position, the relative position of the reflected laser point on the CCD sensor also moves and the distance of the object surface to the reference point can be determined through geometrical relations. Radar system also falls into the category of active sensing. Compared with 
structured light or ToF, the radar-based technology is not affected by lighting conditions.

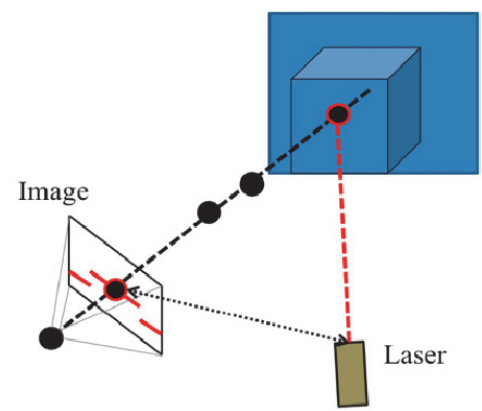

Fig. 7. Illustration of laser triangulation [197].

Contact-less sensing has a wide range of applications in HRC. Wang et al. [254] analysed the sequence of traditional 2D images of human motion in assembly for context-aware recognition. A method to monitor the degradation of industrial robots was introduced by Qiao and Weiss [205], by coordinating different cameras to measure 7D information (time, X, Y, Z, roll, pitch, and yaw). In this research, two main criteria are implemented: (1) the pose accuracy (position and orientation accuracy) of a robot system's tool centre position (TCP), and (2) the ability of a robot system's TCP to remain in position or on-path when loads are applied. Project Soli, a gesture recognition technology based on miniature radar to reach sub-millimetre accuracy for motion detection, is developed by Google. It allows a human hand to become a natural, intuitive interface for a robot [255]. Berri et al. [20] demonstrated the capability of human face tracking as well as gesture recognition using a web camera and a depth camera from Microsoft Kinect, respectively. The depth camera in Kinect uses an infrared projector for active depth sensing [132].

\subsection{Smart sensor network and sensor data fusion}

Different sensing techniques provide different aspects for varying interests [130]. For example, Fig. 8 summarises the sensing techniques for human hand gesture recognition. This section provides an overview of the techniques and a wide range of applications of sensor data fusion and integration reported for HRC, where measurement accuracy and robustness are improved and complex assembly procedures are coordinated.

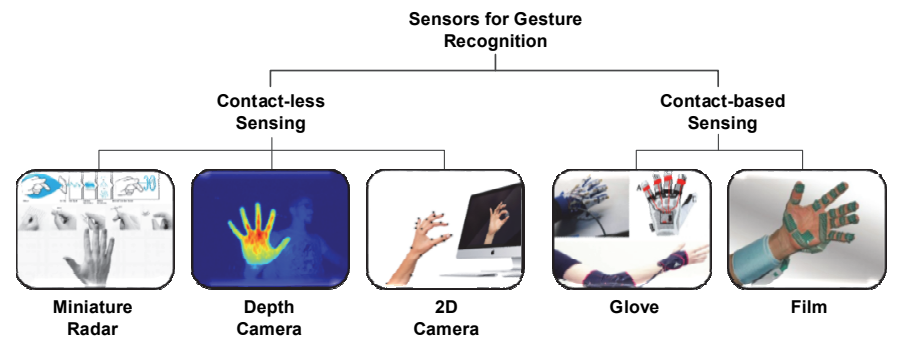

Fig. 8. Multimodal sensors for gesture recognition.

\subsubsection{Localisation, mapping and tracking in $H R C$}

For robots to be truly interactive, they must have the ability to navigate the physical world autonomously to assist human operators. This leads to an application in localisation, mapping and tracking. Probabilistic data fusion methods are generally based on Bayes' rule for combining the prior and the observed information. They provide a means of inferring about an object described by a state, give the observations, and are the dominant techniques used [102,223]. Probabilistic data fusion requires the assumption of conditional independence among the observations. Based on this assumption, the object state update process enables asynchronous sensor data fusion [52]. As the task of localisation, mapping and tracking is most commonly based on a contact-less sensing system, which is subject to occlusions and environmental variation, e.g., changing lighting condition, the probabilistic data fusion provides enhanced robustness to these adverse conditions. For example, the method will still work even if some sensors stop working. Among the probabilistic fusion techniques, Kalman filter (KF) and Particle filter (PF) are the most widely used [223].

The combined localisation and mapping problem has been known as simultaneous localisation and mapping (SLAM), which refers to the simultaneous estimate of both robot and landmark locations, as shown in Fig. 9. Durrant-Whyte and Bailey [52] reported the SLAM solutions based on extended Kalman filter (EKF) and PF using vision system data. Canedo-Rodríguez et al. [31] argued that the SLAM based on vision system alone usually fails when there is not enough geometric variation (such as indoors) and when people are walking around as they cause occlusions. They proposed a PF-based sensor fusion method from data generated using multimodal sensors such as laser rangefinder, $\mathrm{Wi}-\mathrm{Fi}$, cameras and magnetic compass to overcome the limitation. Performance from different combinations of sensors were evaluated. The authors also discussed different aspects of enhancement provided by specific sensor. For example, the compass helps in terms of refining the orientation.

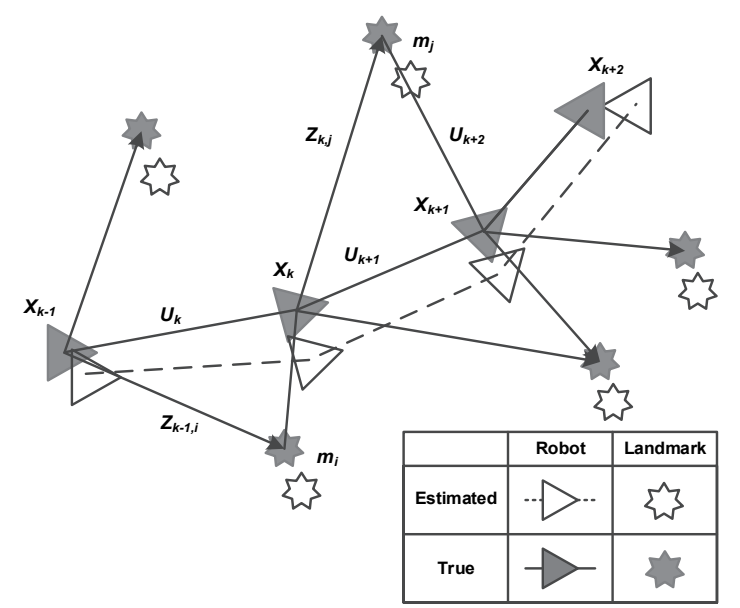

Fig. 9. The SLAM problem. $\boldsymbol{x}$ : robot state vector (i.e., position and orientation); $\boldsymbol{u}$ : control vector; $\boldsymbol{m}$ : landmark location; $\boldsymbol{z}$ : robot observation of landmark location. Subscript indicates time step [52].

An accurate and reliable knowledge of the position and orientation of the robot component, especially the robot arm, is essential to effective operation and human safety. Position and orientation estimation of robot has been another active area where sensor data fusion finds applications. Liu et al. [129] proposed a multi-sensor combination measuring system (MCMS) to improve the pose accuracy of the robot arm. In particular, a closed-loop measurement system was set up. A high precision industrial 3D photogrammetry system was used to dynamically track and measure the robot pose in real time. The photogrammetry system is composed of 4 motion-sensitive CCD cameras set on top of the robot. KF and multi-sensor optimal information fusion algorithm (MOIFA) were investigated in the research to improve accuracy. An up to $78 \%$ improvement in pose accuracy of the robot manipulator was reported. Moreover, a joint-angle estimation method using low-cost inertial sensors was presented by Cantelli et al. [32]. Specifically, three cascaded EKFs have been used to estimate the joint angles by the fusion of the outputs of tri-axial gyroscopes and accelerometers.

As robots are becoming more mobile, the ability to differentiate humans from the environment and being able to track and follow their motion is of paramount importance in HRC. Colombo et al. [44] proposed a wearable device (for the user) with tri-axial accelerometer, gyroscope, magnetometer and a network of external camera nodes to achieve position tracking of the user. 
The user position is measured by cascading two EKFs. Outside the probabilistic method, a less intrusive method was proposed by Knoop et al. [103] for human tracking which fuses 2D and 3D data using the extended Iterative Closest Point approach. The sensors deployed include a colour camera, time-of-flight camera and a laser rangefinder, which were placed on the robot. This method is marker-less and gives complementary information about the tracked body, enabling not only tracking of depth motions but also turning movements. The approach of vision and inertial data fusion was investigated by Martinelli [153], which consists of a monocular camera, three orthogonal accelerometers and orthogonal gyroscopes. A closed-form solution has been derived which expresses the states in terms of the sensor measurements. Other notable alternatives to the probabilistic data fusion include fuzzy logic and Dempster-Shafter method [223].

\subsubsection{Human-robot collaborative assembly}

The complimentary nature of different sensing modalities, such as vision, voice and pressure/force, motivates the consideration of synergistically integrating them for improved effectiveness and efficiency in the advancement of symbiotic HRC assembly towards human-like capabilities. As an example, a vision system allows the robot to gain surrounding information such as environmental geometry and human intention, which is crucial for trajectory and action planning/control as well as collision avoidance. On the other hand, the perception of pressure and force enables compliance to the local constraints, required by specific tasks. This indicates that complex assembly procedures can be coordinated autonomously for enhanced HRC.

One application of sensor integration is the screwing task proposed by Shauri et al. [221], where the trajectory of the robot arm is controlled based on the measurement from the vision system and the robot hand configuration is adjusted based on the pressure/force data. The vision/force integration is also explored in the context of collaborative screw fastening [40], where the data from Kinect, black/white camera and force sensor, deployed to track human hand, screw and contact force, respectively, are used alternately for robot control. De Gea Fernández et al. [62] extended sensor data integration from IMU, RGB-D (red, green, blue, depth) camera and laser scanner to robot whole-body control. The RGB-D and laser scanner are responsible for human tracking while the IMU, integrated into the operator's clothes, recognises the human intention through gestures.

Further application of data fusion/integration in HRC has been reported by GarcÍa et al. [61]. In their research, data from resolvers/encoders, a wrist force/torque $(\mathrm{F} / \mathrm{T})$ sensor and an inertial sensor were fused through a robot tool dynamics model and extended Kalman filter. The goal was to estimate the contact $\mathrm{F} / \mathrm{T}$ and eliminate the effects of non-contact $\mathrm{F} / \mathrm{T}$, such as those produced by inertial and gravitational effects. Koch et al. [104] presented an approach that combines vision, force and acceleration sensor data for contour following tasks (such as machining using an industrial robot). The vision data drives the robot along the workpiece while the force-feedback control maintains the desired contact force, the acceleration sensors are used to compensate the force measurements for inertial forces, and the contact forces between the robot and the environment are used to adjust the measurements from the triangulationbased vision system to compensate for the environmental variation or deformation. Pfitzner et al. [199] fused structured light with a ToF camera for 3D surface mapping of objects by determining the suitable transformation between the two sensors. Héliot and Espiau [78] proposed the fusion of thigh inclination, shank inclination and insoles pressure of human walk for improved phase estimation in cyclic motion using $\mathrm{KF}$ as well as a dynamical system approach. In HRC, the improved phase information can be used for tele-operating robot synchronised with external signals, such as real human walk. A tool for supporting human operators in shared industrial workplaces has also been reported in the form of a software application for wearable devices, such as smartwatches, which provides functionalities for direct interaction with the robot [67]. The results indicate that the approach can significantly enhance the operators' integration in an HRC assembly system.

\section{Active collision avoidance}

\subsection{Safety standards and systems}

Human safety is of upmost importance in any HRC system. Fig. 10 summarised the causes of potential accidents in HRC into three categories: (1) engineering failures, (2) human errors, and (3) poor environmental conditions [33,239]. Engineering failures include the failures of a robot's components. For example, if the sensor detects that the distance between a human operator and a robot can be hazardous, but the control system does not respond properly due to faulty algorithm, it may lead to a collision. Human errors in an accident include design mistakes and unintended interaction errors. Design mistakes are caused by faults or defects introduced during design, construction, or any post-production modifications to a robotic cell. Interaction errors are caused by faults introduced by inadvertent violations of operating procedures. Environment factors refer to extreme temperature, poor sensing in difficult weather or lighting conditions, which is common in vision-based approaches. All of these failures can lead to incorrect response by both the robot and human operator.

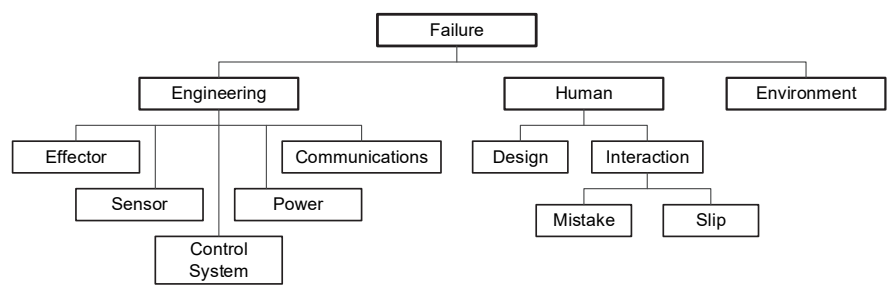

Fig. 10. Taxonomy of failures, adapted from [33] and [239].

Standards and directives aim to standardise the design and prevent engineering failures from the design phase (Tables 2-4). In general, ISO 13855 [86] defined the positioning of safeguards with respect to the approaching speeds of parts of a human body. As one type of machinery, robotic cells shall have the minimum distances to a hazard zone from the detection zone or from actuating devices of safeguards. However, differences between HRC assembly and conventional industrial manipulation requires that safety and reliability standards be rethought [25,215]. Direct contact is inevitable in HRC and the minimum distance and hazard zone need to be re-defined. As a result, ISO/TS 15066 [85] was published to define the biomechanical limits for HRC. The main output is the limit of transferred energy and moving speed.

Table 2. EU directives [163].

\begin{tabular}{l|l}
\hline Title & Description \\
\hline $2006 / 42 / \mathrm{EC}$ & Machinery Directive (MD) \\
\hline $2009 / 104 / \mathrm{EC}$ & Use of Work Equipment Directive \\
\hline $89 / 654 / \mathrm{EC}$ & Workplace Directive \\
\hline $2001 / 95 / \mathrm{EC}$ & Product Safety Directive \\
\hline $2006 / 95 / \mathrm{EC}$ & Low Voltage Directive (LVD) \\
\hline $2004 / 108 / \mathrm{EC}$ & Electromagnetic Compatibility Directive (EMC) \\
\hline
\end{tabular}

Table 3. Indicative general standards [199].

\begin{tabular}{l|l}
\hline Title & Description \\
\hline EN ISO 12100 & $\begin{array}{l}\text { Safety of machinery - General principles for design - Risk } \\
\text { assessment and risk reduction }\end{array}$ \\
\hline EN ISO 13949-1/2 & $\begin{array}{l}\text { Safety of machinery - Safety-related parts of control systems } \\
\text { - Part 1: General principles for design, Part 2: Validation }\end{array}$ \\
\hline EN 60204-1 & $\begin{array}{l}\text { Safety of machinery - Electrical equipment of machines - } \\
\text { Part 1: General requirements }\end{array}$ \\
\hline
\end{tabular}


Table 4. Robot standards [199].

\begin{tabular}{l|l}
\hline Title & Description \\
\hline EN ISO 10218-1 & $\begin{array}{l}\text { Robots and robotic devices - Safety requirements for } \\
\text { industrial robots - Part 1: Robots }\end{array}$ \\
\hline EN ISO 10218-2 & $\begin{array}{l}\text { Robots and robotic devices - Safety requirements for } \\
\text { industrial robots - Part 2: Robot systems and integration }\end{array}$ \\
\hline ISO/PDTS 15066 & Robots and robotic devices - Collaborative robots \\
\hline
\end{tabular}

From the design's perspective, Bdiwi et al. [17] classified the human-robot interaction (HRI) into four levels. In every level, different kinds of safety functions are developed, linked and analysed. Kulic and Croft [115,114] proposed planning and control strategies based on explicit measures of danger during interaction. The level of danger was estimated based on factors influencing the impact force during a human-robot collision, such as the effective robot inertia, the relative velocity and the distance between the robot and the human.

In recent years, many approaches have tackled the safety issues to guarantee the human safety assuming that the physical contact is unavoidable. Michalos et al. $[163,160]$ summarised the robot safety in three categories, i.e., crash safety (only 'safe'/controlled collisions allowed), active safety (stopping the operation in a controlled way), and adaptive safety (intervening in the operation of and applying corrective actions). Based on assembly process specifications, different control, safety and operator support strategies have to be implemented in order for the human safety and the overall system's productivity to be ensured.

Based on the control method, Heinzmann and Zelinsky [77] described the formulation and implementation of a control strategy for robot manipulators which provides quantitative safety guarantees for the user of assistive robots. A control scheme for robot manipulators was developed to restrict the torque commands of a position control algorithm to values that comply to pre-set safety restrictions. Yamada et al. [260] evaluated the human pain tolerance for the purpose of establishing a human safety space. Then they attained velocity reduction on the robot side activated by the incipient contact detection at the surface, and gave the human side an interval margin for the purpose of any reflexive withdrawal motion to avoid more severely interactive situations where the contact goes beyond the limit of safety space. Similarly, Haddadin et al. [69,71, 72] summarised a systematic evaluation of safety in HRI, covering various aspects of significant injury mechanisms. Evaluation of impacts between robot and human arm or chest, up to a maximum robot velocity of $2.7 \mathrm{~m} / \mathrm{s}$, were presented to give the operators a 'safety' feeling [73]. They also approached the safety problem from a medical injury analysis point of view in order to formulate the relation between robot mass, velocity, impact geometry and resulting injury qualified in medical terms [70].

From an energy perspective, Laffranchi et al. [116] presented an energy-based control strategy to be used in robotic systems working closely or cooperating with humans. The presented method bounds the dangerous behaviour of a robot during the first instants of the impact by limiting the energy stored in the system to a maximum imposed value. Meguenani et al. [158] proposed physically meaningful energy related safety indicators for robots sharing their workspace with humans. The kinetic energy of the robotic system and the amount of potential energy that is allowed to be generated within an HRC system during physical contact are utilised, to limit the amount of dissipated energy in case of collision and modulate the contact forces, respectively.

\subsection{HR collision detection}

It is crucial to detect any collision between a robot and a human

operator before any severe accident occurs. Different approaches have been taken by researchers in the past years, many of whom detect the force and contact in real time as they directly relate to a potential collision. Fig. 11 shows the classification of undesired direct contact scenarios between a human and a robot.

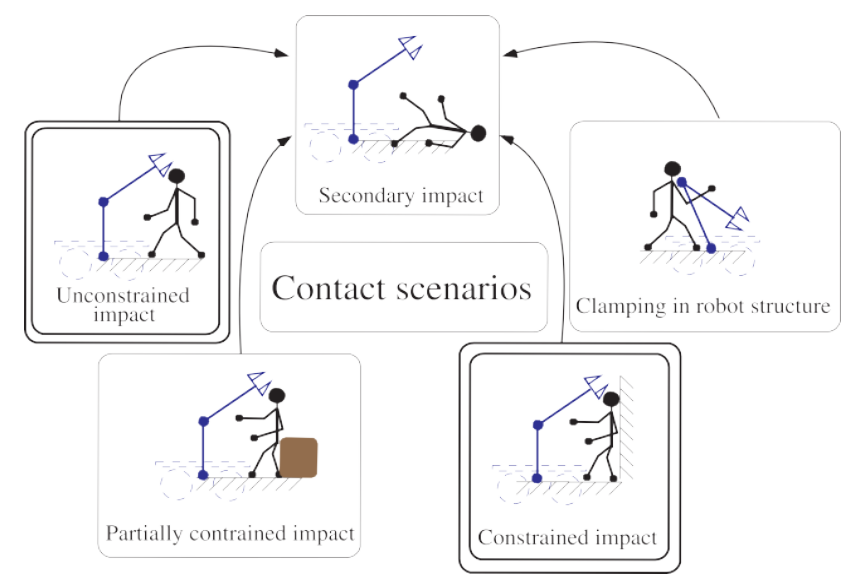

Fig. 11. Classification of undesired contact scenarios between a human and a robot [69].

Some of the research modifies the fundamental robot structure. The Institute of Robotics and Mechatronics of German Aerospace developed a light-weight robot based on the integrated torquecontrolled mechanism [26,137]. The integrated joint torque sensors are deployed in all robotic joints, and potentiometers are added to the common motor position sensors, allowing for the implementation of safety features. Based on variable stiffness actuation (VSA) motors, Tonietti et al. [231] proposed to improve the actuators control in real-time. Both the reference position and the mechanical impedance of the moving parts in the machine are manipulated in such a way to optimise the performance while intrinsically guaranteeing safety. Similarly, Park et al. [190] proposed a safe joint mechanism composed of linear springs and a modified slider-crank mechanism achieved by passive mechanical elements. Geravand et al. [64] developed a closed control architecture to detect the collision based on the outer joint velocity reference to the robot manufacturer's controller, together with the available measurements of motor currents and joint positions. An online processing of the motor currents allows for distinguishing between accidental collisions and intended human-robot contacts, so as to switch the robot to a collaboration mode when needed.

In parallel, the forces applied can be limited by an industrial robot manipulator during contact without the use of external sensors $[105,48,252]$. Using a time-invariant dynamic model in combination with artificial neural networks, the current and torque required by each joint for a given trajectory are estimated with satisfying precision. Focusing on the torque changes at the joints, De Luca et al. [141,143] developed a physical collision detection/reaction method based on a residual signal, and a collision avoidance algorithm based on depth information of the HRC. If a collision takes place, a momentum-based method can apply the reaction torque to the joints, reduce the effective robot inertia seen at the contact, and let the robot safely move away from the collision area $[139,140,142]$. Morinaga and Kosuge [173] proposed a collision detection system based on a nonlinear adaptive impedance control law. The system detects collisions based on the difference between the actual input torque to the manipulator and the reference input torque. The manipulator stops when a collision is detected. Similarly, Lu et al. [138] developed a neural network and model-based method to detect the collision forces and disturbance torques on the joints of a robot manipulator.

Some other researchers utilised machine vision to develop image-based inspection mechanisms to detect potential collisions, 
based on normal 2D cameras. Lin [127] used polyhedrons to model a non-convex object and refined polyhedral approximation for a curved boundary as well, to quickly detect the collision from both convex and non-convex objects. In 2002, Ebert and Henrich [55] presented a collision-detection method based on images taken from several stationary cameras in a work cell. The collision test works entirely based on the images and does not construct a representation of the Cartesian space. Krüger et al. [108] utilised multiple 2D cameras to monitor the workspace, and then calculated a three-dimensional model of the scene. This model is used to determine the spatial distance between the worker and the robot and therefore governs the decision whether to intervene in the control programme of the robot.

In recent years, depth sensor has become a popular approach to detecting the collision between a robot and unknown objects (in most cases, human operators) [34], as it can output the dynamic reflection of objects in 3D models directly. Fischer and Henrich [58] developed a method to detect the minimum distance to any obstacle, which is used to limit the maximum velocity. Flacco et al. [59] developed a fast method to evaluate distances between the robot and possibly moving obstacles (including humans), based on the depth data. The distances are used to generate repulsive vectors that are used to estimate the obstacle velocity and control the robot accordingly.

Combining virtual 3D models of robots and real camera images of operators, an augmented environment can be established to achieve real-time active collision avoidance [251]. Similarly, Morato et al. [172] utilised multiple Kinects to build an explicit model of the human and a roll-out strategy, which can simulate the robot's trajectory in the near future. The real-time replication of the human and robot movements inside a physics-based simulation of the work cell is established, which enables the evaluation of the human-robot separation in a 3D Euclidean space and can be used to generate safe motion goals for the robot.

\subsection{Active collision avoidance}

An HRC environment requires the coexistence of both humans and robots. The consistent safety of humans in such environment is paramount, including both the passive collision detection and active collision avoidance by monitoring human movements and controlling the robots, respectively, to achieve human safety at all-time [218].

Early research on collaborative robots was reported by Bi et al. [22], which was extended with a dynamic control model for better performance by $\mathrm{Bi}$ and Wang [23]. An effective online collision avoidance in an augmented environment, where sensordriven virtual 3D models of robots and real images of human operators from depth cameras are used for monitoring and collision detection $[166,264]$.

Several recent approaches for HRC have also been reported. Argavante et al. [1] and Monje et al. [169] introduced a control system for humanoid robot to carry out a joint operation with an operator. Takata and Hirano [227] presented a solution that adaptively allocates human operators and industrial robots in a shared assembly environment. Bobka et al. [27] developed specialised simulation tools using real-world geometrical data to investigate different algorithms and safety strategies. Chen et al. [37] introduced an optimisation process with multiple objectives based on simulation for assignment and strategy generation of human-robot assembly tasks. Krüger et al. [111] highlighted the merits and available technologies of HRC assembly cells. Using a human-robot shared approach can reveal both the reliability of robots and the adaptability of humans [244]. Anton et al. [6] used the sensor's depth data from the environment and then the processing power of a workstation to detect humans and robots. Using skeleton tracking, a software agent is able to monitor the movements of the human operators and robots, to detect possible collisions, and to stop the robot motion at the right time. Augustsson et al. [11,12] presented an approach to transferring data to the robot communicating the human's position and movements, forcing the robot to respond to the triggers, and visualising the information about the settings and assembly order to the human.

On the other hand, such a system can provoke additional stress to human operators if implemented in poorly designed assembly lines. Therefore, Arai et al. [7] measured an operator's mental strain caused by the location and speed of a robot with respect to the operator, intending to establish a beneficial hybrid assembly environment. Furthermore, Kulic and Croft [113] used robot motion as a stimulus to estimate the human effective state in real time; the developed system analysed the human biological indicators including heart pulse, perspiration level and facial expression.

Several recent approaches attempted to successfully detect and protect operators in locations shared by humans and robots. Two methods were widely considered: (1) using a vision system to perform 3D inspection [112,265] through 3D models as well as skin colour detection to perform 3D tracking of human body in a robotic cell, and (2) inertial sensor-based approach [45] using geometry representation of human operators through a special suit for motion capturing. Real-world experiments indicate that the latter approach may not be considered as a realistic solution as it relies on the existence of a particular uniform with sensing devices and the inadequacy of capturing the movement around the person wearing the uniform, leaving the neighbouring objects unsupervised. This can create a safety leak as there may be a possibility of collision between a moving object and a standingstill operator. More details of varying sensing methods can be found in the literature surveys [21,263].

Among vision-based methods, the efficiency of collision detection has been the motivation for many researchers. For example, Gecks and Henrich [63] implemented a multi-camera collision detection system, whereas a high-speed emergency stop was utilised in the work by Ebert et al. [54] to avoid a collision using a specialised vision chip for tracking. A projector-camera based approach was presented by Vogel et al. [241], which consists of defining a protected zone around the robot by projecting the boundary of the zone. The approach is able to dynamically and visually detect any safety interruption. Tan and Arai [228] reported a triple stereovision system for capturing the motion of a seated operator (upper-body only) by wearing colour markers. Nonetheless, relying on the colour consistency may not be suitable in uneven environmental lighting conditions. In addition, the tracking markers of mobile operators may not appear clearly in the monitored area. Instead of markers, a ToF (time-of-flight) camera was adopted for collision detection [216], and an approach using 3D depth information was proposed by Fischer and Henrich [58] for the same purpose. Using laser scanners in these approaches offers suitable resolution but requires longer computational time, since each pixel or row of the captured scene is processed independently. On the other hand, ToF cameras provide high performance solution for depth images acquisition, but with insufficient level of pixel resolution (capable of reaching $200 \times 200$ ) and with rather high cost. Recently, Rybski et al. [213] acquired data from 3D imaging sensors to construct a three-dimensional grid for locating foreign objects and identifying human operators, robots and background. Ahmad and Plapper [2] also introduced a ToF sensor-based information collection and intelligent decision methodology in order to localise the unknown, un-programmed obstacles and propose a safe peg-in-hole. More recently, an integrated approach for collision avoidance using the depth information from Kinect sensors was reported $[59,60,218]$. Depth image processing for collision avoidance is illustrated in Fig. 12.

In addition, Dániel et al. [46] used both an ultrasonic sensor and an infrared proximity sensor directly on a robotic arm to avoid collisions with industrial considerations, i.e., (1) redundant 
robotic arms, (2) reconfiguration of the robot not with moving the end-effector during avoidance, and (3) automatic stop and warning function when the avoidance is impossible without moving the end-effector. Moreover, other researchers like Cherubini et al. [39] incorporated both F/T sensors and vision systems into a hybrid assembly environment to provide a direct interaction between a human and a robot with safety protection.
Camera 1
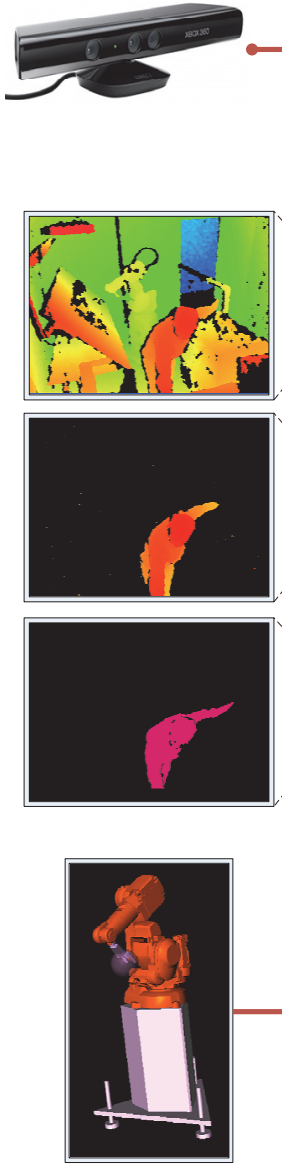

Camera 2
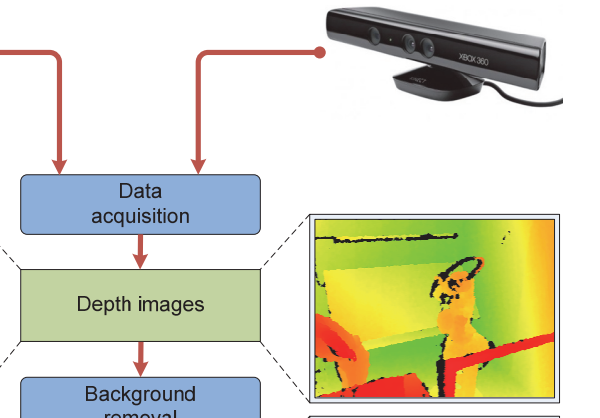

$\downarrow$

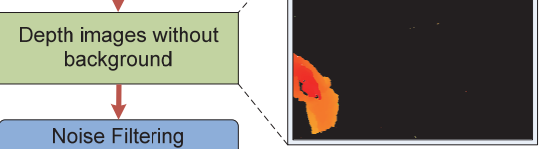

Noise Filtering nd assigning of labels $\downarrow$

Detected operator

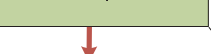

$3 \mathrm{D}$ visualisation $\downarrow$

\section{$3 \mathrm{D}$ point cloud of} detected operator

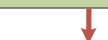

Introducing the 3D model of robot Detecting and avoiding any collision

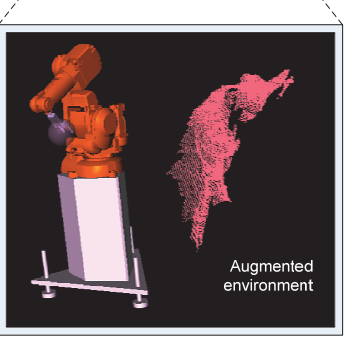

Fig. 12. Procedures and outcomes of depth image processing, adapted from [218].

Calinon et al. [30] proposed an active control strategy based on task space control with variable stiffness, and combined it with a safety strategy for tasks requiring humans to move in the vicinity of robots. A risk indicator for human-robot collision is also defined, which modulates a repulsive force distorting the spatial and temporal characteristics of the movement according to the task constraints.

Sensor data can be used for programming a robot's motion and controlling the program's execution in a fenceless setup [151], as shown in Fig. 13. Safety is ensured with the use of 3D sensing devices, while the tasks' coordination is managed by the so-called station controller. The programming approach combines both offline and online methods, in an intuitive manner. Schlegl et al. [217] proposed a sensor and control method that mimics the behaviour of whiskers by means of capacitive sensors to achieve short response time. After the installation of capacitive proximity sensors, robots can sense when they approach a human (or an object) and react before they actually collide. Osada and Yano [187] proposed a novel collision avoidance method for mealassisting robot with a SwissRanger SR3000 3D camera under a dynamic environment. Similar to an assembly environment, the potential map using diffusion equation is employed to control the behaviour of the manipulator to avoid collision between the manipulator and an obstacle or a user.
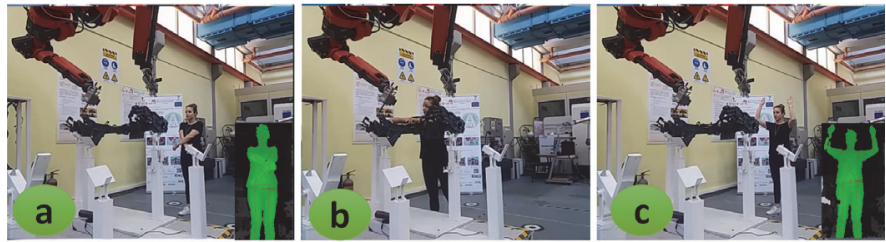

Fig. 13. (a) Human signals the start of a task, (b) human task execution, and (c) human ends the task [151].

In addition, other researchers focused on combining different sensing techniques to track humans and robots on shop floors $[46,207,135,212,157,259]$ which used both ultrasonic and infrared proximity sensors to establish a collision-free robotic environment. Among commercial systems of safety protection solutions, SafetyEYE ${ }^{\circledR}$ [200] of Pilz is a popular choice. It computes $2 \frac{1}{2} \mathrm{D}$ data of a monitored region using a single stereo image and detects violation of predefined safety zones. Accessing into any of the safety zones would trigger an emergency stop of the monitored environment. However, these safety zones cannot be updated during robotic operations.

\section{Dynamic task planning}

\subsection{Context awareness and resource monitoring}

Assembly tasks shared by humans and robots in HRC assembly are dynamic in nature [253]. They are often planned for and assigned to available and capable resources (humans and robots) at the time of collaboration [188]. This requires constant resource monitoring for better context awareness.

Lee and Rhee [121] developed a context-aware 3D visualisation and collaboration platform in three layers as shown in Fig. 14. The context layer maintains contexts from various resources. It facilitates reasoning and execution of those contexts for providing context-aware services. The interface layer supports interactions between physical devices (or software modules) and the context layer. Thus, all the devices and services can be easily registered, searched, and executed. The service layer provides various taskrelated services, e.g., augmented reality (AR) based visualisation, collaboration services, and pre- and post-augmented services considering the contexts. On the other hand, resource monitoring is supported by sensors, 3D models, point clouds and remote computing resources [23,166,245,247,248,250,258,214].

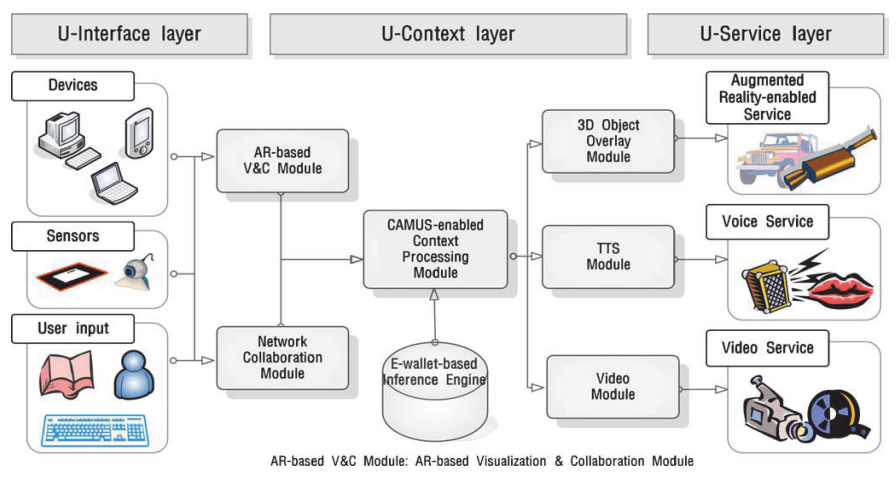

Fig. 14. Context-aware information framework [121]. 
Within the context, an efficient HRC system should be able to understand a human operator's intention and assist the operator during assembly [208]. Since the operator's (work-related) motions are limited and repetitive, an assembly task can be modelled as a sequence of human motions. Existing human motion recognition techniques can then be applied to recognise the human motions associated with an assembly task. Mainprice and Berenson [146] categorised human actions through the use of Gaussian Mixture Models (GMMs) and Regression (GMR). During task execution, the category that best fits the real movements of the human is selected and used as a predictor of the human movements. This prediction is finally considered in order to generate the optimal robot trajectory. In parallel, Liu and Wang [133] modelled the recognised human motions in a Hidden Markov model (HMM). A motion transition probability matrix is then generated after solving the HMM. Based on the result, human motion prediction becomes possible. The human intention is analysed with the input of predicted human motion, which can be used as input for assistive robot motion planning. The industrial robot can thus be controlled to support and collaborate with the human based on the planned robot motions. The workflow of human motion prediction in HRC is shown in Fig. 15.

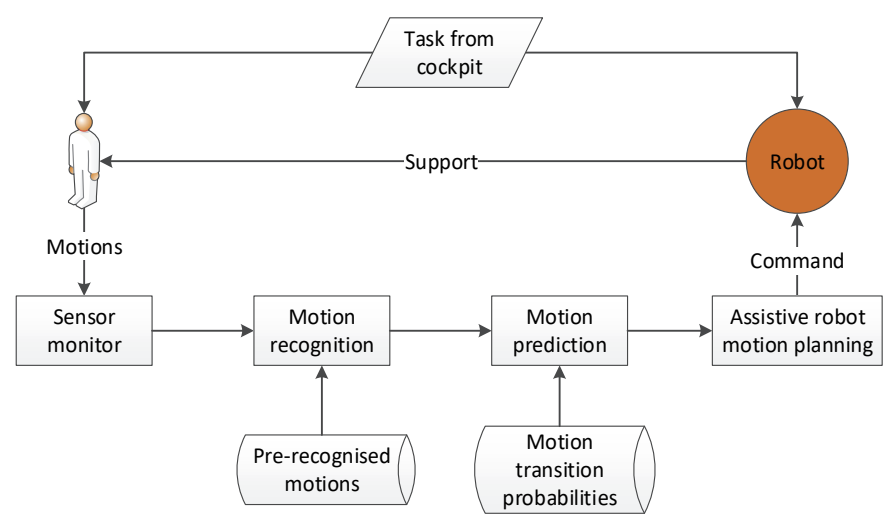

Fig. 15. Workflow of human motion prediction in HRC [133].
Recently, deep learning has gained attentions as a reliable and practical method for human motions recognition and prediction for timely context awareness [14,208,131]. Visual observation of humans' motion provides informative clues about the specific tasks to be performed, thus can be used to establish reliable context awareness. Wang et al. [254] investigated deep learning as a data-driven technique for continuous human motion analysis and prediction, leading to improved robot planning and control in accomplishing a shared task. Fig. 16 shows the architecture of a convolutional neural network for human motion recognition and prediction. An engine case study was carried out to validate the feasibility of the proposed method, as shown in Fig. 17.

\subsection{Dynamic assembly planning}

\subsubsection{Task planning and scheduling}

In HRC assembly like ROBO-PARTNER [162], the focus is given to combining robot strength, velocity, predictability, repeatability and precision with human intelligence and skills to achieve a hybrid solution that facilitates the safe cooperation of operators with adaptive robotic systems. As part of assembly planning, task planning and scheduling is essential for on-demand assembly operations (Fig. 18).

The aim of task planning and scheduling is to allocate and dispatch the tasks to be performed and required by the assembly process to the available resources (e.g., workers, machines and robots), so that the assembly operations are optimised according to a given criterion (e.g., time and energy consumption [167]). Several constraints have to be considered, such as the ability of the resources to perform a task, the availability of all the necessary tools, the time required by each of the resource for performing the task $[227,95,242,183,28,175]$.

In symbiotic HRC assembly, real-time planning and scheduling play a key role in the generation of a plan and its robust execution [186]. Indeed, in comparison to the planning and scheduling problems of fully automated systems $[184,230]$, the presence of the human in the loop introduces a temporal and controllable uncertainty. On one hand, the time required to execute a task by a
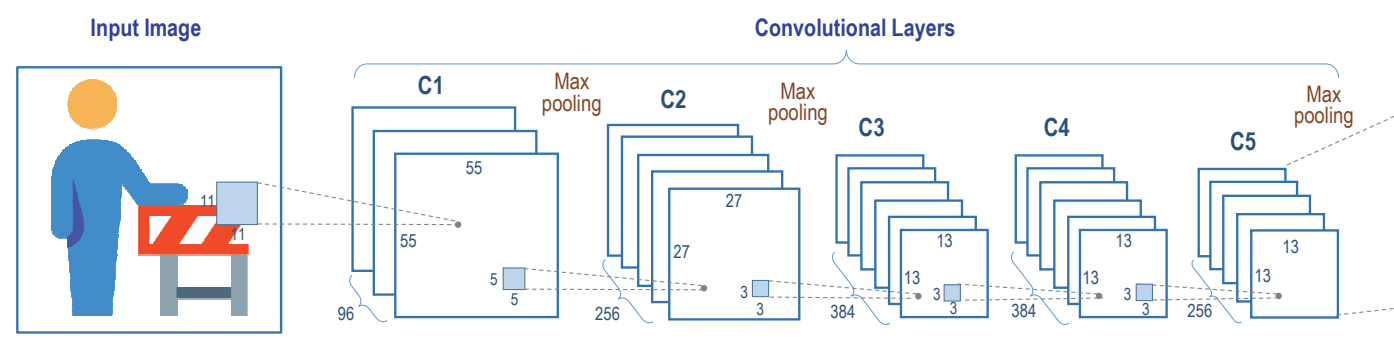

Fully Connected Layers Classified Category

Fig. 16. Deep learning for human motion recognition and prediction [254].
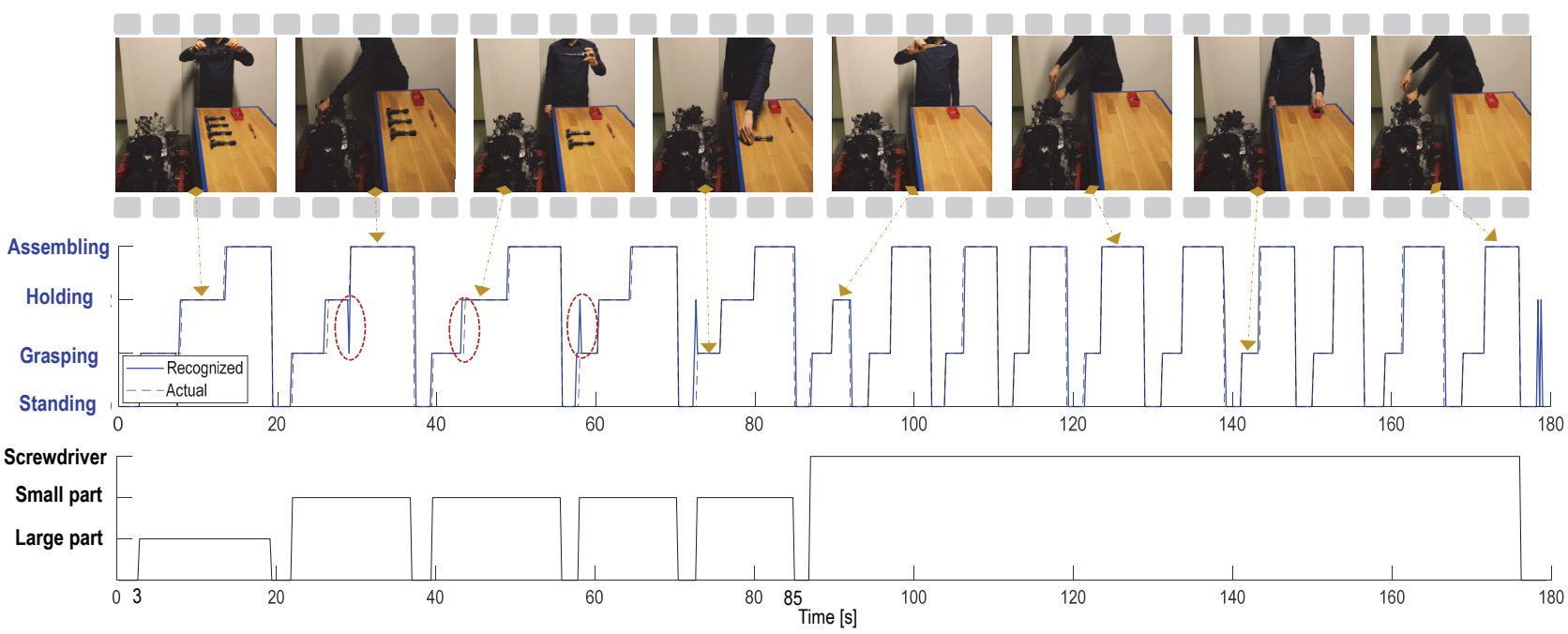

Fig. 17. Sample video frames (top); sequence of recognised human motions (middle); sequence of identified objects (bottom) [254]. 
human is not predefined and depends on the expertise of the worker, on his/her state of health, attention and fatigue. On the other hand, the presence of the worker in the process together with the need for his/her safety, comfort and ergonomics may require a continuous and dynamic adjustment of the assigned tasks. The adjustments, small or relevant on the basis of the specific situation, can lead to the assignment of a new task to the robot (e.g., the posture assumed by a worker in the execution of a task may make the goal of the robot reachable or unreachable to perform a second simultaneous task) or to the modification of robot task trajectory (e.g., the robot path and/or the robot speed have to be modified to avoid a collision or make the human worker more comfortable).

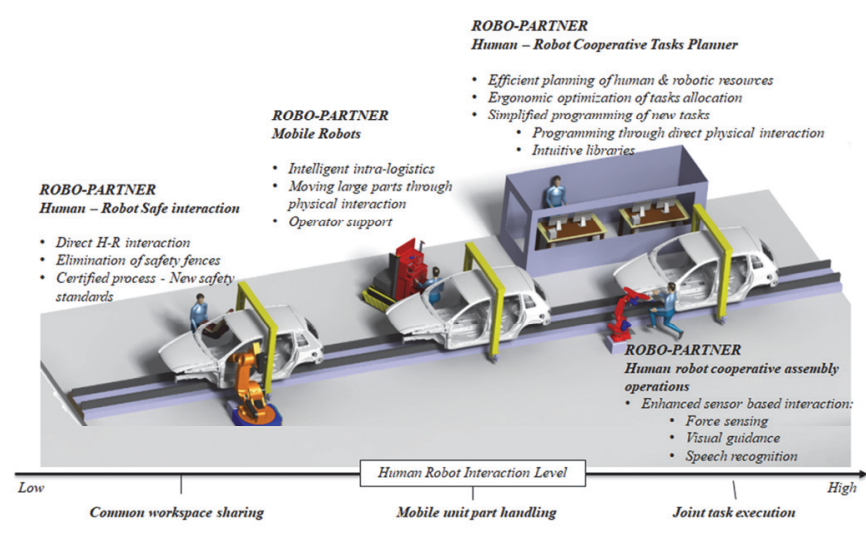

Fig. 18. ROBO-PARTNER in automotive industry [162].

In order to cope with the presence of human in the loop, the task plan is generally constructed at an abstract, high and discrete level and continuously evaluated to decide how and when to execute a planned task, considering temporal/causal constraints, spatial/geometric constraints and controllable/uncontrollable activities. The definition of the plan can rely on Planning Domain Definition Language (PDDL) $[155,65,128]$ or on timeline planning approaches $[176,66,16]$. PDDL is the standard encoding language for 'classical' planning tasks, based on a domain - containing predicates and actions for world definition - and on a set of objects - describing initial state and goal specification. Timeline planning is based on the use of a set of timelines. Each timeline is characterised by a sequence of states, i.e., temporally extended predicates consisting of a proposition and a list of parameters, i.e., start, end, and duration times. States are associated with temporal intervals (with given lower and upper bounds), instead of exact temporal occurrences. Relationships among the timelines are managed by a set of domain rules (also known as compatibilities) that incorporate explicit constraints on the parameters of the states. Despite PDDL-based methods, timelinebased approaches [122,204,15] rely on an explicit representation of time and its temporal constraints, allowing the simultaneous resolution of planning and scheduling problems while taking into account coordinated and synchronous tasks as well as temporal flexibility and uncertainty.

Even if based on timelines, the majority of the planners and schedulers available in the literature today are unable to manage uncontrollable events and/or humans without strongly relying on re-planning mechanisms, thus showing limited applicability in HRC applications. Controllability issues, initially investigated by Vidal [240], have been further investigated by others through the generation of dispatchable execution approaches [53,174]. A hierarchical timeline-based planning approach was developed as a general-purpose planning framework called EPSL (extensible planning and scheduling library) [154,186]. The framework models a human as a planned variable where all values are uncontrollable, thus getting closer to the optimisation of the planning and scheduling problems in HRC. This approach was extended by taking into account and optimising robot motion planning strategies [196].

\subsubsection{Robot motion planning}

In the last decade, a large part of the literature has focused on how to generate robot trajectories for human-robot collaborative tasks. In such a context, literature may be divided into two main groups: online motion planning and offline motion planning [222]. In offline motion planning, a robot trajectory is defined offline or just before its execution, thus requiring possible online adjustments in terms of speed, acceleration or path in order to guarantee human safety and avoid possible dynamic obstacles in the scene. In online motion planning, the robot is instructed with runtime commands heavily depending on the current situation (e.g., a locally optimal robot speed or desired goal is evaluated and then sent to the robot).

Among offline methodologies, Mainprice et al. [147] worked on the automatic generation of robot paths when the robot has to hand an object over to a human. A robot trajectory is planned on the basis of a map representing human's visibility, human's arm comfort and human-robot distance with the idea that the robot has to stay as visible as possible and as far from the human as possible, and that the goal has to be in a comfortable position to be reached by the human. Pandey and Alami [189] presented another approach based on the generation of maps. The method is to identify an optimal robot trajectory considering two maps representing both human and robot perceived reachability and visibility.

Among the online planning methodologies, Lasota et al. [120] proposed the use of a Markov Decision Process (MDP), where human actions and the process of human decision making are modelled as a stochastic transition function influencing real-time robot actions and states. Similarly, McGhan et al. [156] adopted an MDP to model unsynchronised human-robot collaborative but independent tasks (i.e., the human and the robot have to work on different pre-allocated tasks sharing the same workspace). They proposed the adoption of two MDPs: the first MDP is used for predicting the human behaviour, and the second MDP is used to determine the robot action. The use of a Partially Observable Markov Decision Process (POMDP) was proposed by Karami et al. [98] in order to determine the best robot action, when the belief state is represented by human's intention. The human and the robot actions are unsynchronised, i.e., the human and the robot have to work in the same workspace on different uncorrelated goals. Extending the applicability to a higher number of human possible tasks and to industrial robots, Pellegrinelli et al. [195] introduced a framework for human-robot workspace sharing (unsynchronised tasks) based on hindsight optimisation [92]. The approach defines a distribution probability on human's goal on the basis of the movements of the human's hand. This distribution probability is used by a POMDP to define the belief state of the robot and to select the best robot action (i.e., twist).

When dealing with HRC assembly tasks, offline and online approaches for robot trajectory generation present advantages and disadvantages. On one hand, online generated trajectories are more flexible than offline generated trajectories and able to easily take into account variations in the environment and in the human behaviour. On the other hand, the obtained trajectories may have smoothness problems and can lead to frequent robot failures (generally due to high-exerted force/accelerations). Moreover, the estimation of the robot execution time represents a critical issue currently limiting the applicability of these techniques in real industrial contexts for which the task time is a relevant constraint. Indeed, the continuous definition of robot actions, and the continuous re-planning of robot paths and/or speed make it hard to be aware of the time required by the robot to execute the assigned task. This information is however of great interest to optimally plan and schedule assembly tasks. A first attempt to solve this issue was proposed by Pellegrinelli et al. $[195,196]$, where the robot execution time is estimated on the basis of statistical methods taking into account human behaviours and a predefined robot path. 


\subsection{On-demand job dispatching}

Tsarouchi et al. [233] proposed a method for task planning in a hybrid assembly cell which includes both humans and robots. A model is structured addressing in a unified manner both humans and robots as the cell's resources. The sequence of the human and robot tasks is structured in three levels (Fig. 19). The evaluation of the alternative human and robot task plans is based on multicriteria, such as average resource utilisation, mean flow time, and ergonomics. The proposed method was implemented into a graphical software tool and applied to an automotive case study, comprising a dual arm robot and a human operator.

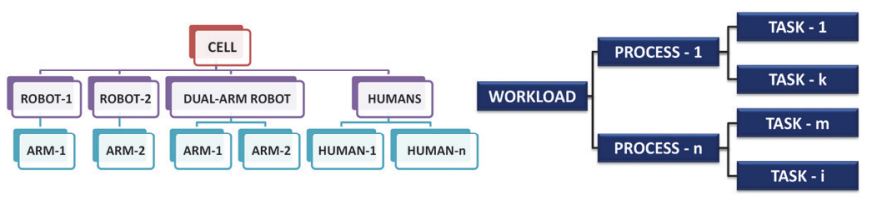

Fig. 19. Hybrid cell model and workload model [233].

Job rotation is the common practice in industry. It provides employees with a more engaging work environment, resulting in far less monotonous and repetitive tasks. A dynamic job rotation tool would allow for the efficient allocation of assembly tasks to suitable operators, at any point of time, leading to more balanced workload distribution and thus, achieving soft 'dynamic line balancing'. A hierarchical approach to multi-criteria and decisionmaking algorithms is used for the implementation of the tool. The tool can generate alternative rotation schedules and evaluates them against predefined criteria [161].

Makris et al. [148] discussed the distribution of information in real time using pushlets/comet technology. In addition, RFID (radio-frequency identification) based identification techniques are used to track products and operators on the shop floor in real time. This identification triggers the automatic transmission of assembly instructions and multimedia materials to handheld or stationary terminals, reducing the time required to retrieve and assimilate the information. They also discussed the architecture design and the implementation aspects of a pushlet-based wireless information environment. The system was validated in a truck assembly line as shown in Fig. 20.

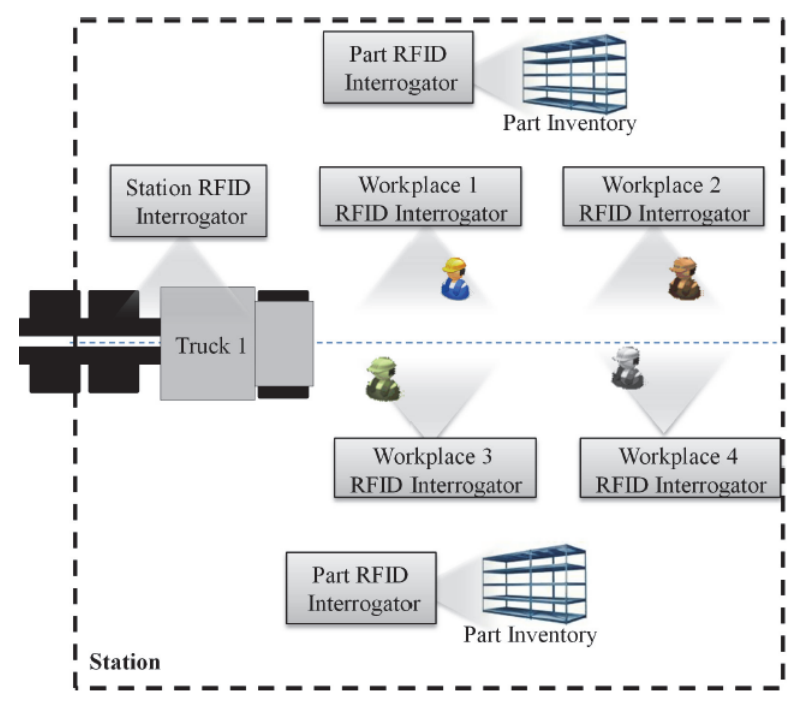

Fig. 20. RFID enabled assembly station [148].

Tsarouchi et al. [233,235] reported a method for the coordination of assembly tasks requiring the cooperation of humans with a robot. A ROS-based architecture is used. The assembly sequence of these tasks and their characteristics were modelled in a neutral XML format generated offline. As shown in Fig. 21, a ROS-based system was developed for different modules to communicate and coordinate their actions through message exchange. The human is able to review the past and upcoming tasks in a graphical user interface. For the execution of the assembly tasks, the Process Simulate tool [224] is used.

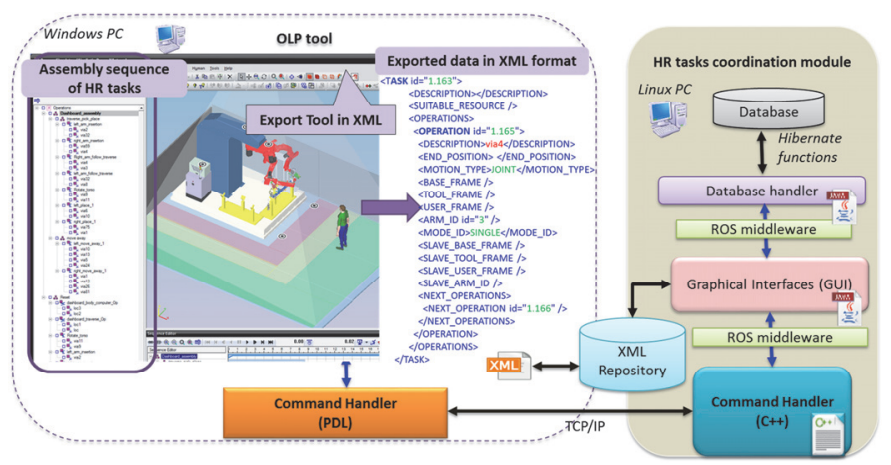

Fig. 21. Software system architecture [235].

\section{Adaptive robot control}

\subsection{Multimodal programming}

The high effort for conventional (re)programming of industrial robots (online and offline) in relation to the decreasing lot sizes of customised production motivates the development of robotic programming and control with higher degree of adaptability. In the recent decades, different scientific approaches have been followed in order to integrate intuitive forms of programming into robot control based on modular software architectures.

Especially for SMEs, robot programming and the requirement of expert knowledge for robot reprogramming is one of the major obstacles for the use of robots in industrial applications. Manual online programming, as the major programming method, often turns out to be a bottleneck. Typically, training courses for the programming of industrial robots take from several days up to several weeks [47].

In the teach-in mode, the approach of the targeted pose represents an intuitive process of demonstration, although the need to press buttons on an operator panel is an obstacle. Furthermore, the reached positions finally need to be integrated in a textual programme sequence. Play-back programming offers a more direct approach of interaction through haptic guidance of the robot. The automatic programme generation based on the demonstration process leads to a further significant simplification of programming. A practical disadvantage is however the limited transferability of this programming method to robot systems with large workspaces.

The programming paradigm 'Programming by Demonstration' (PbD) pursues a comprehensive approach to make the programming of industrial robots more intuitive. This refers to learning by imitation with the aim of transferring human capabilities to the robot. In the meantime, this is a broad field of research that often involves the fields of artificial intelligence, image processing, path planning and motor control in addition to human-robot interaction. At the heart of $\mathrm{PbD}$ is the sensorial observation (perception) of actions that are usually performed by a human user. Based on this information, it is attempted to automatically derive a task or a programme for the robot which imitates certain behaviours of the action being taken.

Multimodal intuitive programming offers opportunities for robot programming without specialised expert knowledge. Multimodality means the use of diverse communication channels between human and robot at the same time. Today, this plays an essential role for the design of natural human-robot interaction and control systems. Different publications [192,19,164,99] have shown that multimodal design of industrial control and programming systems increases productivity. Precondition for this is the application oriented design of the systems. Multimodal control systems combine, for example, gestures (finger, hand, touch), speech and different forms of feedback. 
In the project MORPHA, results were achieved between the years 2000 and 2003, which showed to be fundamental for numerous subsequent research activities in the field of intuitive control and programming for industrial and service robots with basic programming systems based on speech [211] and touch instructions [68,3]. In the project SMErobot [179], speech and gesture based instructions were used for intuitive programming of industrial robots. Also, haptic guidance of the robots was applied for applications in gluing and path welding [262]. In an experimental setup, Vogl [243] showed that based on a pointer device in combination with AR, the programming time could be reduced by over 30\% compared to teach-in methods [209].

In numerous research projects, gestures are applied as a form of nonverbal human communication. According to Pavlovic et al. [192], gestures describe intended movements, usually the arms and hands, with a manipulative or communicative character (Fig. 22). Manipulative gestures are used to manipulate objects (e.g., translation, rotation, deformation). Communicative gestures include a communicative purpose, that is, communicative intent. Information is transmitted to a recipient at different levels. The gesture-based exchange of information can be considered at the pragmatic level (linguistic action), semantic level (meaning of the signs) and syntactic level (signs and rules). In a natural HRC environment, the communicative gestures are typically accompanied by speech. Furthermore, communicative gestures can be subdivided into symbols or actions. The symbols have a linguistic function, which can either have referential character, for example, by the imputation of an object, or a modal function, for example, for the symbolic extension of a linguistic statement. Gestures whose intention has a direct connection to the movement itself can be considered as action gestures. Mimetic gestures call for the imitation of the suggested action, whereby deictic gestures (pointing gestures) represent a local reference to the content of the action.

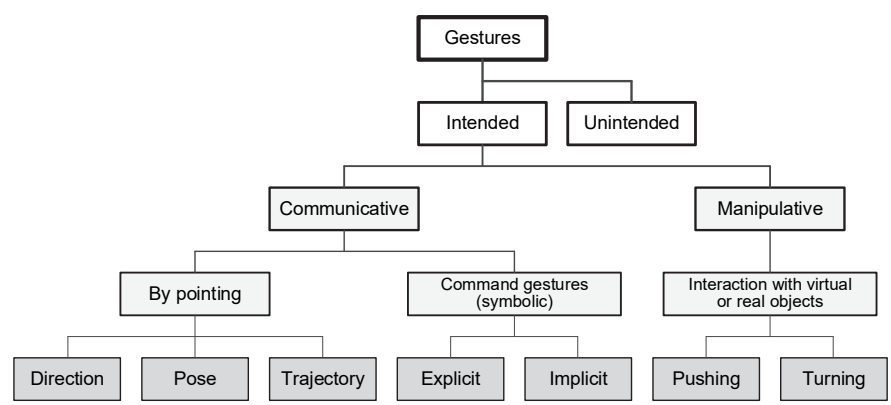

Fig. 22. Taxonomy of gestures in control and programming of industrial robots (adapted from [192]).

Based on the classification of hand gestures according to Pavlovic et al. [192], Fig. 22 shows general applications of the gesture-based input for controlling and programming industrial robots. Main applications of communicative gestures are commands for the motion guidance of the robot. In the case of motion guidance, a distinction should be made in terms of concept and content between manual motion control and manual motion specification.

- Motion control: The robot movements occur simultaneously with the movement of the user. The definition of trajectories or poses is relative, starting from the current position.

- Motion specification: The user defines the spatial parameters of the robot program absolutely. For this, the user is located in the workspace of the robot. The transfer of the movements to the robot takes place after the interaction has ended.

As a further application scenario, control commands can be derived from the gesture-based manipulation of objects. In the following, the suitability of the various applications of hand gestures in the spatial programming system is examined in more detail. A detailed discussion of various marker-less gesture-based interaction principles in robot programming can be found in the literature [117].

Ong et al. [185,42] used a mobile AR application based on an HMD to spatially define trajectories via a handheld marker. This marker is tracked via the camera of the HMD or an external stereo camera system. In addition to the visualisation of the trajectories, the approach includes a kinematic simulation of the trajectories by a virtual robot, which is placed over a real robot. The presented programming system is in principle mobile and can therefore be used as a process-oriented programming method. The definition of trajectories via gesture-based modelling is made possible. This approach describes an intuitive, natural way of defining trajectories, albeit using the marker as an artificial tool. The presented system is, however, limited to the definition of trajectories. Spatial manipulation of the paths as well as a more complex programme management and interfaces to common industrial robot manufacturers are not considered. Although a transfer of the gesture-based definition of trajectories to the field of application of orbital welding [41] was discussed, in practice it is not pursued. Task-oriented spatial programming is also not considered.

Lambrecht and Krüger [118] introduced a method for spatial robot programming by hand and finger gestures based on a stationary 2.5D sensor in combination with a mobile 2D camera in a handheld device. This approach allows adaptation to defined robot trajectories by drawing single points with finger gestures in an augmented reality environment (Fig. 23).

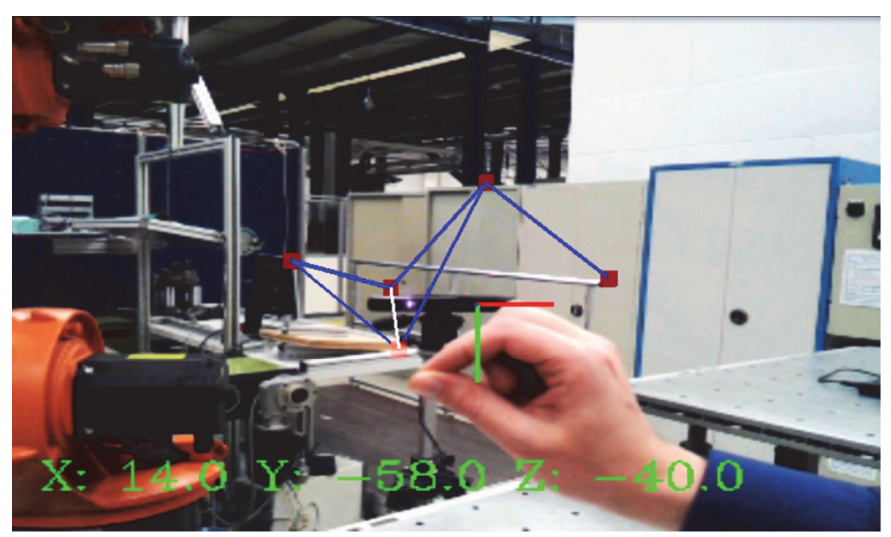

Fig. 23. Pose adaptation for intuitive spatial robot programming by hand/ finger gestures [118].

In the playback method, the robot is haptically guided by the operator. The control system records individual points or entire trajectories via the axis coordinates. To flexibly guide the robot, force-moment sensors and a corresponding compliance control are needed. If these are not available on the addressed robot system, movements can also be recorded via an additional programming or phantom device and be transferred to the addressed robot. A typical application is the programming of painting robots. In master-slave programming, movements are recorded by passing an alternative robot system and transmitted to the target system. Due to the similarity of the method, this programming method can be assigned to the playback method. Beside playback programming, the haptic guidance of a robot by the human can be applied for programming-free robot control.

Lightweight collaborative robots, such as those that have come to the market for assembly applications in recent years [110], allow human haptic guidance [82,35], in particular, to enable intuitive and efficient teach-in of individual poses or playback of movement profiles. Due to the low load-bearing capacity, they are not intended to relieve the physical burden on humans in the context of collaborative task execution [219]. Humanoid dual-arm lightweight robots often utilise playback recording for dual-arm 
operations. As there are no offline programming environments available for industrial dual-arm robots yet, intuitive online programming techniques such as play-back or teach-in offer alternative approaches. Surdilovic et al. [226] proposed an objectoriented programming approach to simplify dual-arm robot compliance programming by formulating bimanual actions which can be used for assembly operations such as BI-Approach, BIHold or BI-Insert-Extract in a natural way. Makris et al. [152] extended this approach to a hierarchical model, which maps bimanual operations to different assembly steps and levels to support a designer to identify options for automation of assembly operations.

Fig. 24 shows a method for intuitive programming of a dualarm robot [152]. A task-oriented programming procedure is applied, including a dual-arm robotics library. The robotics library represents human-like capabilities and implements bimanual operations. This intuitive programming framework is based on a service-oriented system architecture and developed in ROS. The user can easily interact with a dual-arm robot through depth sensors, noise cancelling microphones and GUIs [107].
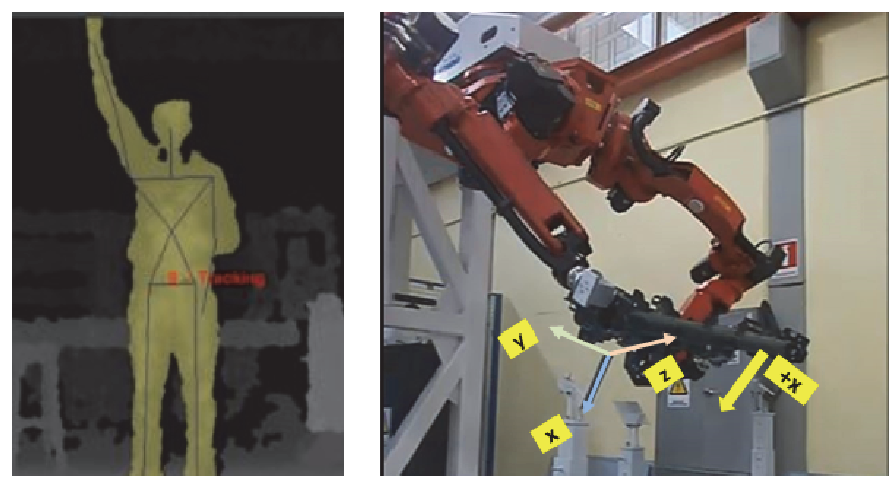

Fig. 24. Human gesture UP for moving away from loading bank (left); a dual-arm robot moves up (right) [152].

In the coming years, robot programming software tools are expected to be more intuitive and user friendly. Tsarouchi et al. [232] proposed a method for simplifying robot programming using visual sensors detecting the human motions. A vocabulary of body and hand gestures was defined, allowing the movement of robot in different directions. An external controller application was used for the transformation between human and robot motions. On the robot side, a decoder application was developed translating the human messages into robot motions. The method was integrated in an open environment based on ROS, enabling the easy extensibility with new functionalities.

\subsection{Smart algorithms embedding}

Intuitive programming of robots based on modalities, such as gestures, speech and haptics, requires embedding of complex algorithms with different levels of abstraction. Typically, a sophisticated function such as recognition of a pointing gesture by a robot, is composed of various sub-functions, e.g., image processing, pattern recognition and coordinate transformation. The underlying system architecture for embedding smart algorithms has a significant impact on the effort for integration and implementation of high-level functions for intuitive robot programming and symbiotic collaboration between humans and robots. In the last two decades, different architecture models have been proposed in order to structure the complexity of software for robot control on different levels. Based on the socalled 5C's principle of separation of concerns [29,203], which separates the communication, computation, coordination, configuration and composition aspects of software functionality, Vanthienen et al. [238] developed a methodology for programming of complex robot tasks called instantaneous Task
Specification and estimation using Constraints (iTaSC). Task specification is also the focus of the LightRocks framework [49].

ROS provides a common framework for the development of robot control including complex functionality from image processing to pattern recognition [206]. Makris et al. [152] propose a service-oriented architecture based on ROS for user interaction with a dual-arm robot. The multimodal interaction is based on gestures, voice commands and graphical user interfaces. In this concept, a hierarchical model decomposes different specific activities in the 'order', 'job', 'task' and 'operations' levels.

One of the essential benefits of the ROS framework is the high reusability of the software. Though, like other frameworks mentioned before, it only provides support for developers. With the focus on the support of end-users, Andersen et al. [5] propose a system-independent execution framework for adaptive and interactive robotic applications. Their concept, the DTI Robot CoWorker (RCW) is oriented to the creation of agile robots, which can easily be reconfigured for new tasks. The concept was evaluated for complex industrial assembly and handling scenarios. A general overview about robotic frameworks and architectures was given by Kortenkamp and Simmons [106].

Another approach to algorithm embedding is the use of function blocks (FBs) [249]. FBs are described in IEC 61499 [83], as an IEC standard for distributed industrial processes and control systems, particularly for PLC control. It is based on an explicit event-driven model and provides for data flow and finite state automata-based control. According to IEC 61499, FBs may exist in different types. The definition of an FB describes the external interface and internal behaviour of a particular type of FBs. Fig. 25 shows the external structure of a basic FB and a composite FB. A basic FB defines the fundamental functional relationships of events and data, from which a composite FB can be built. It uses an appropriate language (such as Structured Text or Java) to define its states and algorithms. The algorithms are encapsulated inside the basic FB and can be accessed by the basic FB itself only. A composite FB may consist of several basic and/or composite FBs connected by events and data, but the composite FB itself does not contain any algorithms.

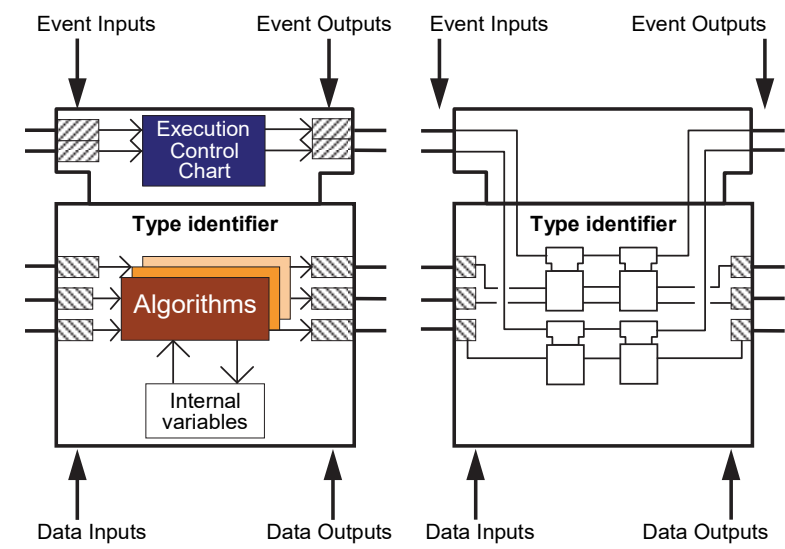

Fig. 25. Function block structures: a basic FB (left) and a composite FB (right).

Wang et al. [246] defined the mating relationships in assembly as basic assembly features (e.g., inserting, riveting, press fitting, and screwing), and mapped the assembly features to basic FBs. Thus, a complex assembly process can be decomposed into the assembly features and handled by FBs for the ease of robot control. FB-embedded algorithms enable online runtime decisionmaking for trajectory planning and motion control. For example, Fig. 26 presents (a) the graphical definition of an inserting $\mathrm{FB}$, and (b) its execution control chart (ECC). The graphical definition gives the details of the inputs/outputs of events and data, as well as embedded algorithms and variables. The ECC is a finite state machine, which specifies the transitions from the events to their corresponding algorithms for event-driven execution. 


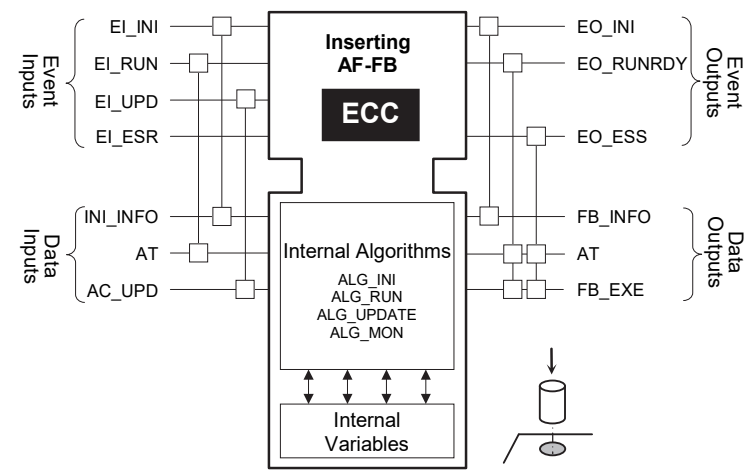

(a) Graphical definition of an inserting FB

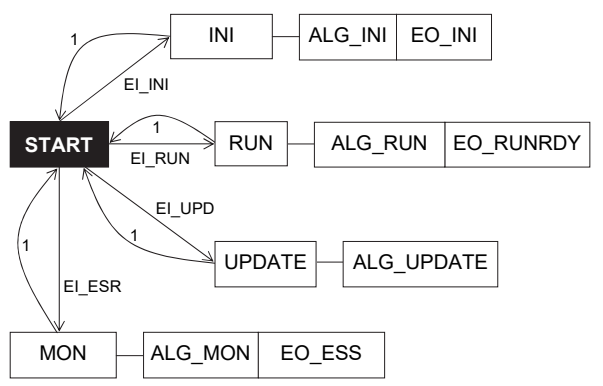

(b) Execution control chart of the AF-FB

Fig. 26. Graphical design of an inserting FB and its ECC [246].

During robotic assembly, the insertion task is decomposed into two steps: picking up a pin and inserting the pin into a hole, as shown in Fig. 27, along a series of positions of the end-effector of a robot. How to accomplish the insertion task is purely driven by the embedded algorithms based on environmental data.

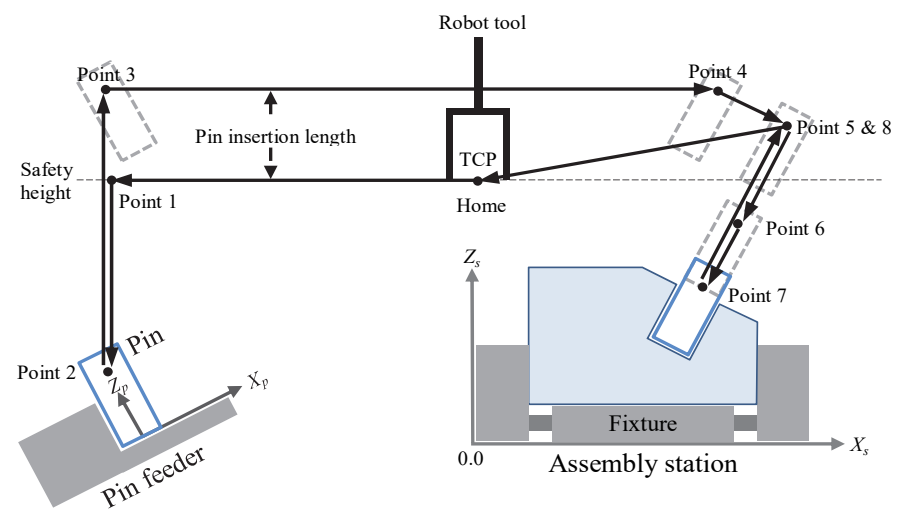

Fig. 27. Step-wise procedure of pin insertion by FBs [246].

\subsection{Programming-free robot control}

While mostly a robot is programmed for repetitive tasks, there are various applications in assembly where high flexibility needs either control without programming or auto-adaptation of tasks by the robot without explicit reprogramming by a human.

A very intuitive form of symbiotic HRC can be achieved by the direct haptic guidance of the robot by an operator. This mode can be used not only for online programming scenarios such as teaching or programming by demonstration [8], but also for direct collaborative workpiece handling in assembly.

In recent years, larger robots for haptic collaboration with humans have been introduced, whose construction aims at symbiotic collaboration in such a way that a human brings in his/her superior cognitive and sensorimotor capabilities and the robot contributes with its mechanical power in order to reduce the physical strain of the human worker. The basis is the control algorithms, which ensure both the safety of the human in the haptic connection with the robot, and at the same time allows an efficient support of the human by the robot [109,225]. The control system is based on sensor systems integrated in the robot, which enable the robot to record the control parameters specified by the operator. The main control parameters for the haptic coupling are 6-DOF force and torque signals, from which variables for position, velocity and acceleration of the robot are derived in the control. Beside haptic guidance of a robot, other modalities for instruction of the robot were also developed, including speech and gestures $[3,68,179,211,229]$. With respect to automatic adaptation of the robot to changing tasks and environmental parameters, methods and algorithms from the field of machine learning show a high potential. Levine et al. [123] demonstrated the high potential of deep learning algorithms for automated flexible robotic grasping of different objects in undefined poses. Maeda et al. [144] proposed a method to achieve fast and fluid human-robot interaction by estimating the progress of the movement of the human. Their method (Fig. 28) allows the progress, or the phase of movement, to be estimated even when observations of the human are partial and occluded; a problem typically found when using motion capture systems in cluttered environments. It can generate a corresponding robot trajectory before the human finishes his/her movement.

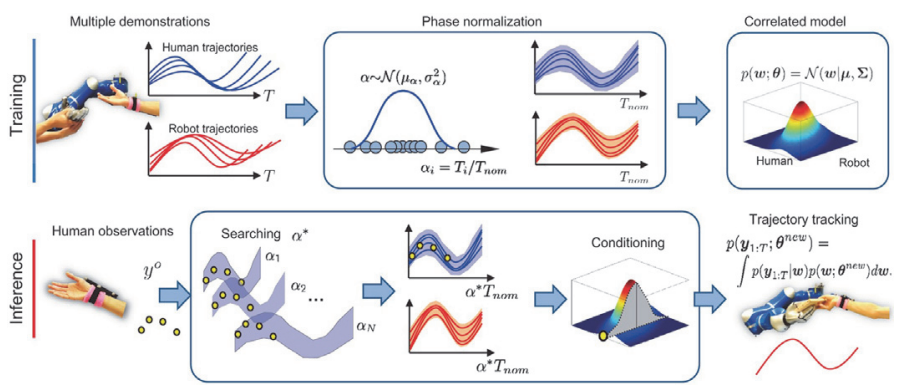

Fig. 28. Action recognition and trajectory generation [145].

\subsection{Brainwave-driven robot control}

Brainwave-driven robotics, or brain robotics, is a fast emerging field that will positively impact symbiotic HRC assembly in the near future. In brain robotics, electroencephalography (EEG) is used to analyse and understand the behaviours of human brains, the results of which are used to communicate with robots and control the robots directly. Recently, Mohammed and Wang [168] proposed using human brainwaves as a means for robot control in HRC assembly, where an Emotive Epoc+ device was chosen as the EEG measuring headset to record brain activities (Fig. 29).

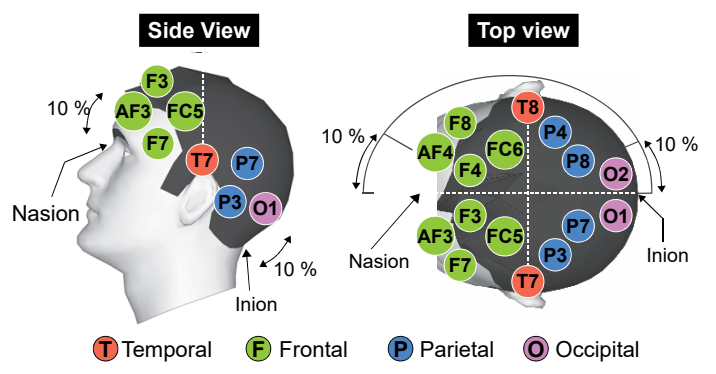

Fig. 29. Locations of electrodes of an Emotive Epoc+ device [168].

In order to retrieve a stable and true mental command from a human operator, a set of preparatory training sessions is needed. After proper training, the mental command in the form of multichannel brainwave patterns can be recorded, which is then used for robot control. Fig. 30 illustrates the framework of brainwavedriven robot control for HRC assembly. At the control level, an FB triggered by the mental command is used to generate a trajectory together with other control parameters, and move the robot for programming-free control.

Two major advantages were reported in their research work: (1) it frees the hands of an operator, allowing the operator to 
control a robot while performing a related task shared with the robot, and (2) it provides an auxiliary channel for multimodal symbiotic HRC assembly in addition to voice, gesture and haptic commands. Using mental commands can overcome the difficulties in noisy environment when voice commands are used alone. The challenge is the reliability of the mental commands.

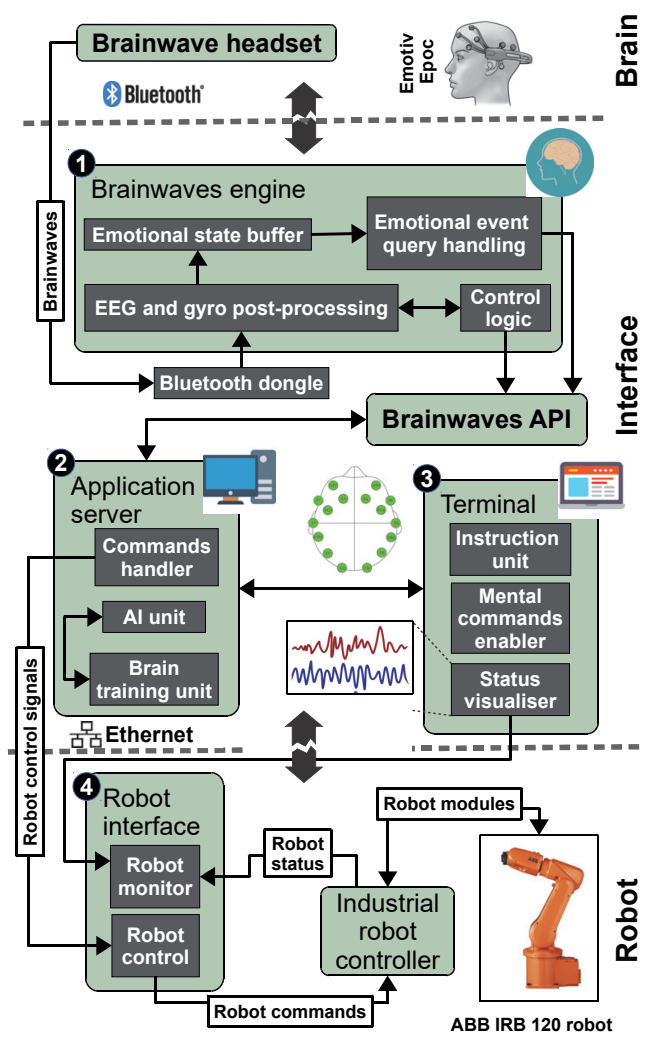

Fig. 30. Brainwave-driven robot control [168].

\section{Mobile worker assistance}

\subsection{Mobile worker tracking and identification}

In HRC assembly, the environment is mostly structured except humans and mobile objects. 3D models can be used to represent the structured environment, whereas the humans and the mobile objects can be captured by vision/motion sensors [218]. As shown in Fig. 31, physical sensors are linked to a collision avoidance system to drive the behaviour of the 3D models and monitor the human in real time. By reading the joint values of the robot from its controller, the present pose of the robot can be introduced to the HRC environment and visualised using the 3D models. At the same time the human can be tracked and represented as a point cloud with the help of vision sensors.

In their research, Kinect sensors are employed for surveillance of unstructured foreign objects in the robotic cell, including the operator who lacks the representation in the 3D space. To sustain the rapid processing, the closest range between the 3D model of the robot and the point cloud of the human is used to detect any collision in an augmented environment. With the calculated relative distance between the two, a suitable decision can be made to actively control the robot so as to effectively avoid a possible collision. In order to track a human operator for safety reasons, three Kinect sensors can be used to eliminate blind spots in the shared environment, as shown in Fig. 32. For worker identification, a quick response (QR) code is used, which is affixed to the worker's uniform or helmet. Alternatively, an RFID chip can be embedded in the worker's ID.

Fig. 33 illustrates one view of a user sharing its workspace with a KUKA LWR4 manipulator, with the experimental setup used to track and detect the human operator and control the robot in a closed loop [158]. The system tracks the human using two Kinect sensors. The distance between the human and the robot is used to constrain the kinetic energy of the robot so as to limit the amount of dissipated energy in case of a collision. The amount of potential energy that is allowed to be generated in the HRC system during physical contact is used to modulate the contact forces.

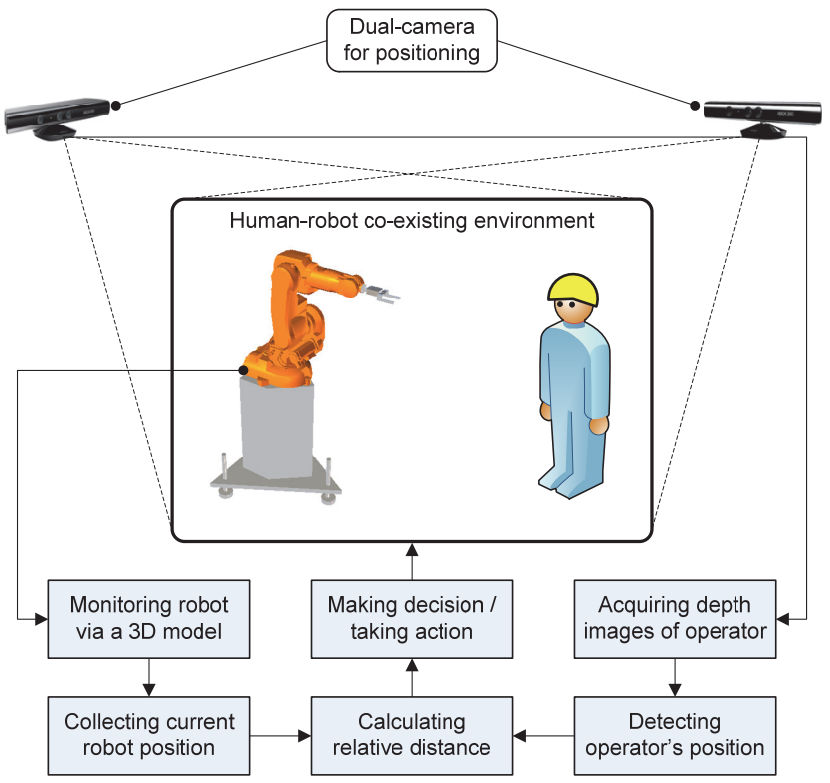

Fig. 31. Mobile worker tracking in augmented environment [218].

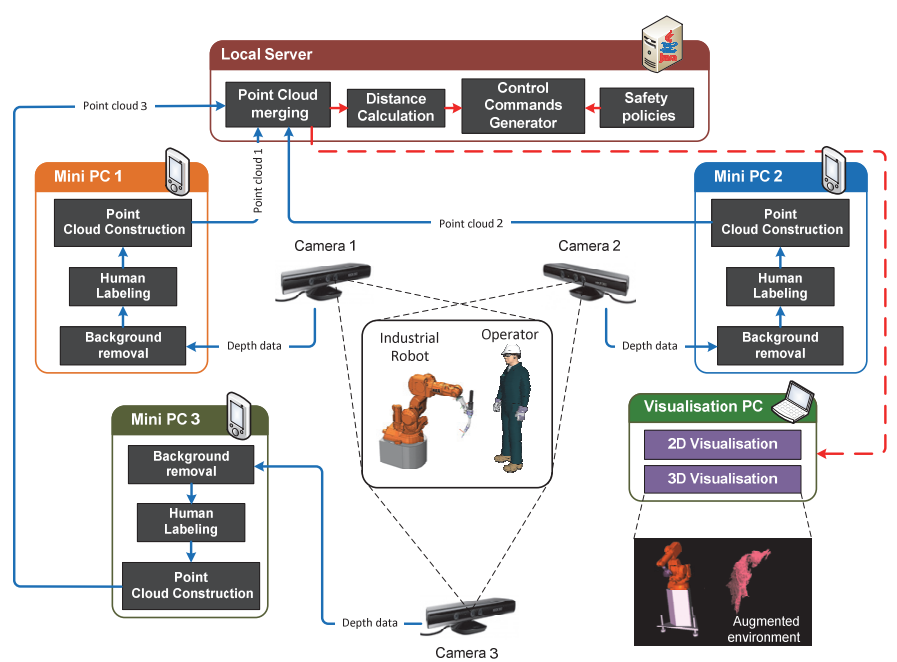

Fig. 32. Mobile worker tracking by three Kinect sensors

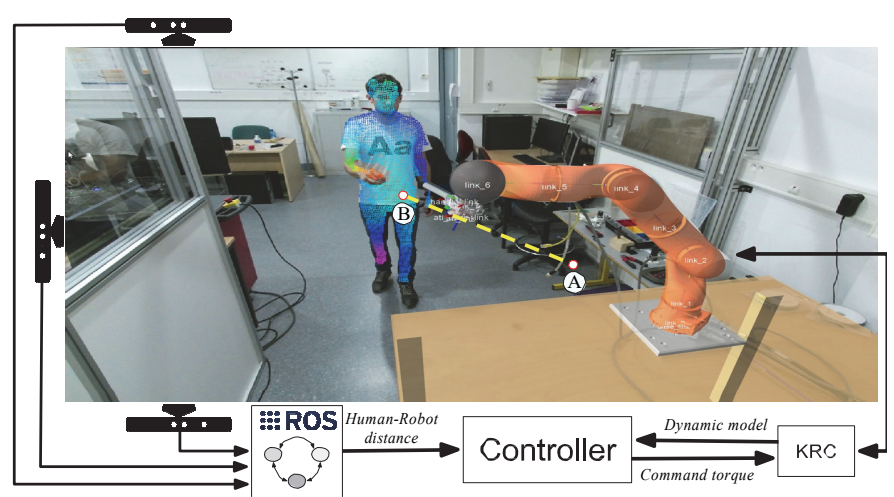

Fig. 33. Human tracking and detection in robotic environment [158].

\subsection{AR-based in-situ decision support}

Existing and evolving trends and paradigms in manufacturing, such as mass customisation and personalisation, call for better communication among product design and production execution. 
Specifically, the lack of information feedback from and to shop floors can lead to lower product quality and increased production times. In state-of-the-art industrial practices, the most common visual interface devices for communication comprise control unit terminals, TFT monitors mounted over workstations, as well as the growing trend of mobile PCs and tablets. New technologies, such as AR, have also been considered in academic research for decision support in process simulation, operator guidance, and training.

To be useful, a decision-support system should provide instant and intuitive in-situ support to human workers based on realtime information feedback on shop floors in the HRC assembly context. AR technologies have been found instrumental to this requirement. According to Azuma [13], an AR system shall possess three characteristics: (1) the system should combine real and virtual worlds; (2) the system is interactive in real time; and (3) the system is registered in three dimensions. In HRC assembly, an AR system enables intuitive graphical display which allows a human worker to understand the assembly tasks immediately so as to prevent assembly errors.

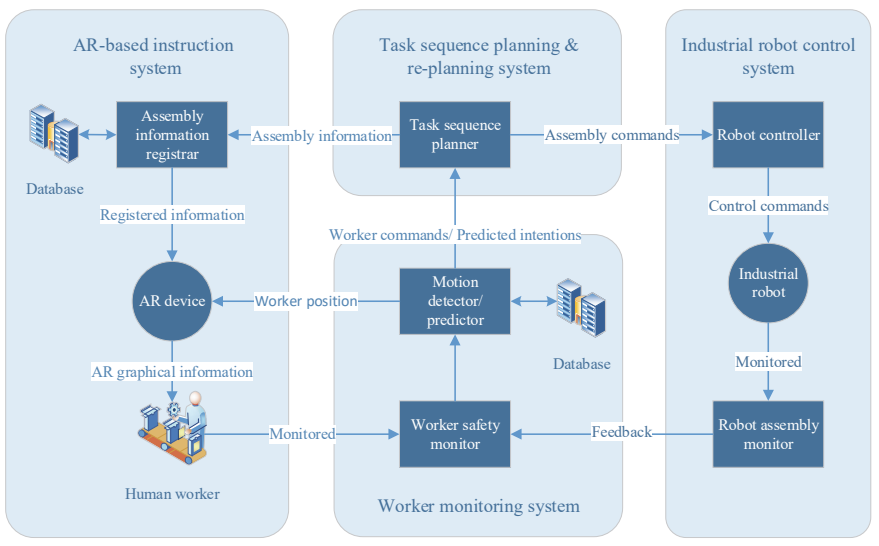

Fig. 34. Architecture of an AR-based worker support system [134].

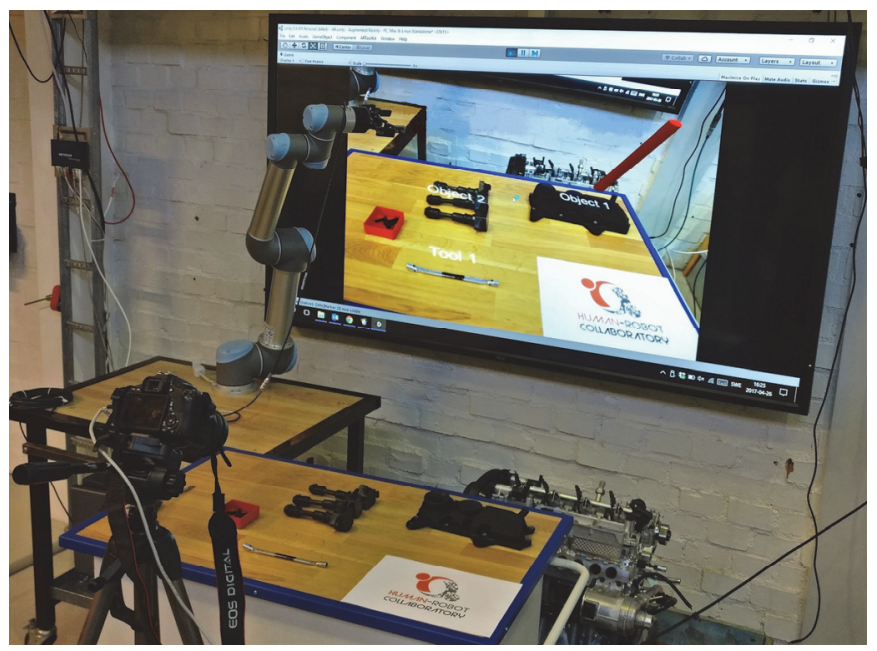

Fig. 35. An example of AR-based in-situ worker support.

Fig. 34 shows the system architecture of one AR-based in-situ decision-support system [134], consisting of four sub-systems: AR-based instruction system, task sequence planning \& replanning system, worker monitoring system, and industrial robot control system. The AR-based instruction system is responsible for in-situ support to workers by receiving and delivering assembly instructions in the HRC context. Assembly instructions are placed at the correct location at the right time when the corresponding assembly parts are detected in the real-world coordinate system. The detection is achieved by comparing the real assembly parts captured by the vision sensor with the corresponding ones saved in a database. An AR device of any type receives the registered graphical information and displays the information according to the real-world coordinate system. Finally, a human worker performs the assembly task with the support of the AR-based graphical information (Fig. 35).

Another tool reported by Gkournelos et al. [67] is to support operators in shared HRC workplaces. The tool was developed in the form of a software application for wearable devices, such as smartwatches in Fig. 36. It provides functionalities for direct interaction with a paired robot. Interfaces to audio commands, manual guidance applications, and AR visualisation systems were implemented. The development of the smartwatch application was carried out in the ROS framework.
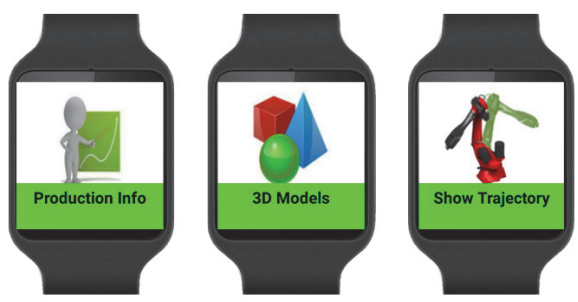

Fig. 36. Interfaces for AR functionalities [67].

To aid operators in HRC assembly, Makris et al. [149] designed and implemented an AR tool to provide production and process related information as well as to enhance the operators' immersion in a safe HRC environment. The developed system has been integrated with a service-based station controller, which is responsible for orchestrating the flow of information to the operators, according to the task execution status.

The role of the AR-based system reported by Michalos et al. [159] is to provide multimodal support, e.g., AR, video and textbased instructions and assembly status updates. The system is also aimed at enhancing the operator safety and HRC acceptance through the immersion capabilities of AR. Real-time visualisation of robot trajectory as well as the actively monitored areas where the robot is allowed/forbidden to enter are indicative examples. A hardware landscape including AR equipment and markers, handheld devices for user input and network infrastructure for interfacing the robot with a storage database was provided. The software architecture for coordinating the AR system with the assembly process and the data retrieval from the robot controller was also presented. The tool has been tested on a pilot assembly case of a rear axle as shown in Fig. 37.

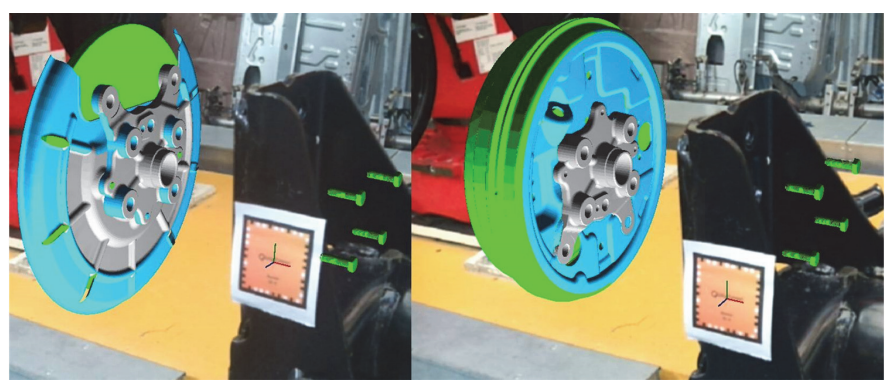

Fig. 37. Multi-modal visualisation of assembly models [159].

AR technologies are also applied to robot path planning and trajectory planning to arrive at collision-free solutions $[178,38]$. Fig. 38 demonstrates one example of user-guided planning of the orientation of a robot end-effector, resulting in a collision-free geometric path on the target surface.

Despite the aforementioned AR applications, how to combine AR with simulation has remained as a challenge for many years. This challenge was tackled in final assembly operations [150]. This study adopted an assembly sequence generation algorithm for the creation of assembly sequences and steps, and carried out by engineers with the use of CAD data and any additional input concerning a given product. This information is then delivered to 
an AR module, which is responsible for the visual instructions of the assembly task. The AR module is based on an algorithm that creates the virtual instructions as the base assembly sequence of a specific product. The instructions are stored in the form of a template. Fig. 39 highlights the instructions of assembling a cap to the casing of a differential.
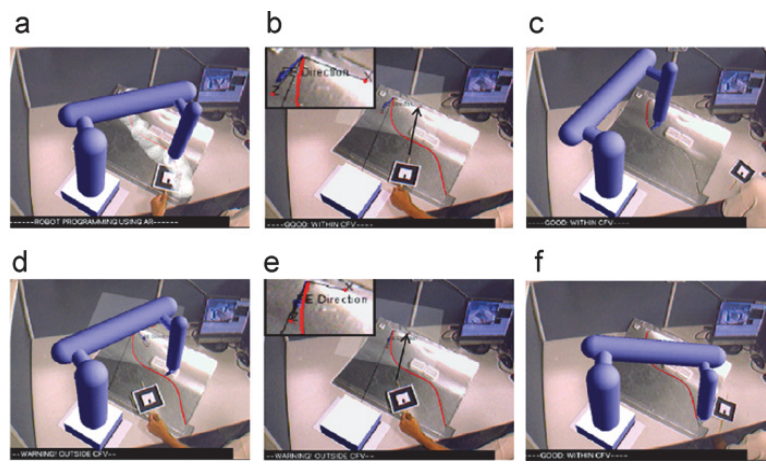

Fig. 38. AR-based collision-free path planning [178]

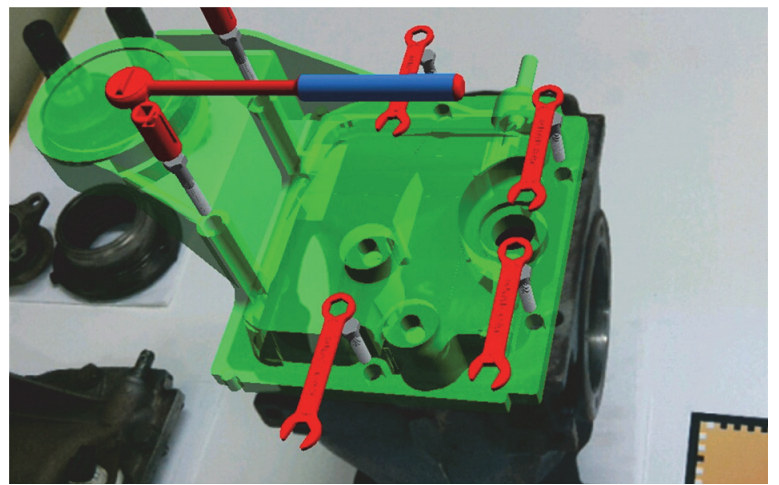

Fig. 39. Virtual instructions for finalising the assembly of a cap to the casing of a differential [150].

Pintzos et al. [201] proposed a novel use of AR goggles, coupled with other mobile devices for communication with people, working on shop floors and in engineering offices. The proposed methodology tries to address the challenges, related to the use of both technologies (and their respective interfaces) by presenting an integrated approach: the use of a mobile device as an input device and as a fiducial marker for the positioning of a virtual screen in front of the user. After the presentation of the concept, its advantages and disadvantages, compared with current practices as well as with the rest of the relevant academic work, were presented. The technical implementation to be realised, including specific software frameworks that will be used was also described. Special attention is given to the data models that will support the implementation of this approach and to show how they can be integrated into the existing systems and practices. Fig. 40 shows how information is delivered on a monitoring screen over a handheld device.

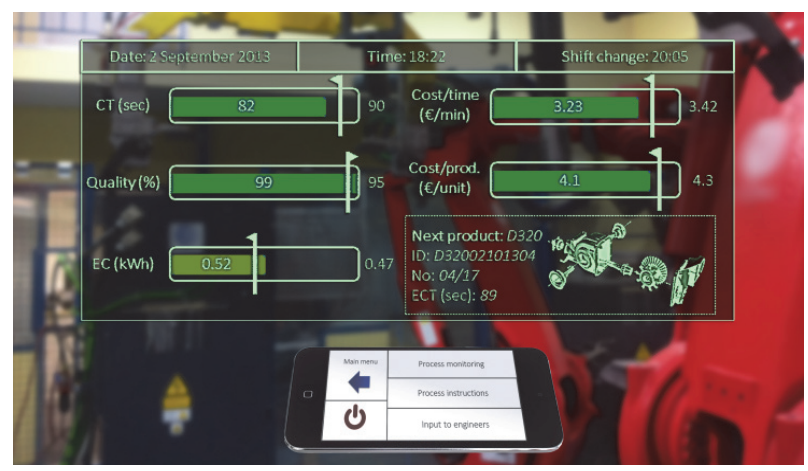

Fig. 40. Monitoring screen over a handheld device [201].
AR can help close the gap between product development and manufacturing operation, mainly because of its ability to reuse and reproduce digital information and knowledge intuitively, while supporting assembly operators. For example, a semanticbased AR system [210] was reported for integrating existing information and knowledge available in CAD/PDM systems, and for supporting human operators in real time. This study is supported by an algorithm used for generating AR instructions based on the product and process semantics. The results are demonstrated in an automotive use case as shown in Fig. 41.

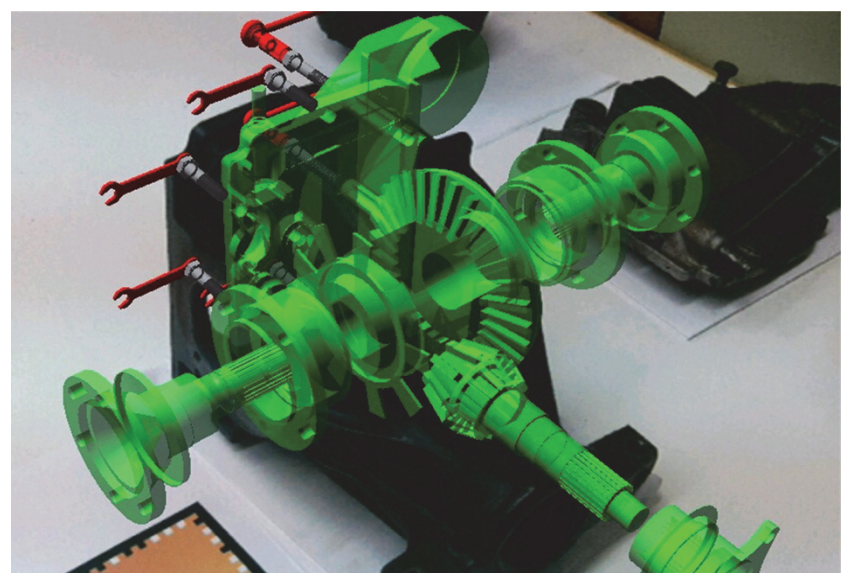

Fig. 41. Full assembly visualisation [210]

\subsection{Ergonomic and psychological challenges}

\subsubsection{Aspects of ergonomics}

Collaborative robots are currently used in assembly as human companions and helpers, making the human and the robot a team $[76,18,39,193,198]$. In any case, the way in which a robot performs an assigned task, i.e., the way in which it moves and operates, substantially affects the final performance of the whole human-robot team. Specifically, the robot(s) should change its/their behaviours to make the human comfortable and to increase his/her ergonomics performance, while keeping low the time necessary to the team to perform all the tasks. An essential aspect of HRC is how to cope with human ergonomics, process time, emotions and reaction during collaboration, and safety aspects.

The assessment of ergonomic performance metrics of an HRC setting can be performed by virtual manufacturing tools which are equipped with digital human models for the simulation of manufacturing tasks. For instance, the ErgoToolkit system [4] implements ergonomic analysis methods, already available in the literature or in industrial practice, into a state-of-the-art virtual manufacturing software. The particular ergonomic tools handle posture definition and recognition, as well as stress screening. The former provides the functionality of defining and automatically recognising digital human postures, while the latter evaluates any unfavourable situations in a manual work process. It is shown that they are viable tools in detecting ergonomic problems, early in a development process, prior to physical installation. The study concludes with presenting the application of the tools in an automotive case study (see Fig. 42). Such a virtual commissioning is also applied to a flexible assembly cell, which includes a dual-arm robot in cooperation with humans for assembly tasks typically performed by operators [151].

Ergonomics involves not only physical but also mental stress whereas monotony and other forms of "underload" are to be avoided on the one hand, just like "overload" (fatigue and burnout due to persisting overstrain and lacking degrees of freedom in choices, pace, transparency, etc.) on the other hand. While the former is clearly alleviated by HRC, handling overload in a collaborative robotic work environment calls for psychological considerations. 


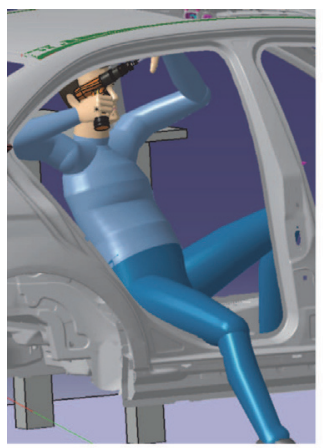

(a)

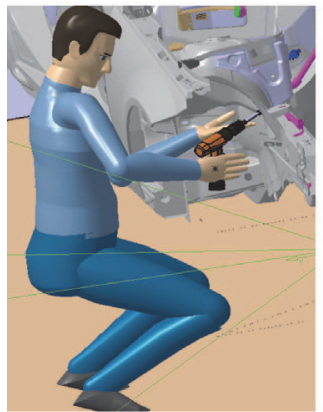

(c)

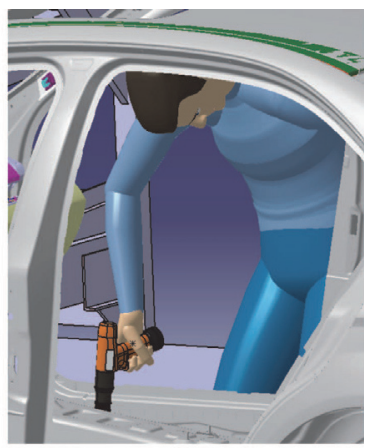

(b)

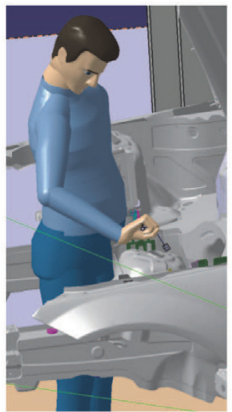

(d)
Fig. 42. Sequence of assembly operations of a car (snapshot from DELMIA V5 simulation) [4].

\subsubsection{Psychological challenges in human-robot collaboration}

In order to understand how to improve the efficiency of a human-robot team, several papers focused on the identification of a set parameters of the robot trajectory that influence human behaviour. Analysing the robot's movement, relevant factors to be considered are the legibility and predictability of the robot's trajectory [51] which can result in the correct identification of the final goal of the robot on the basis of its initial trajectory (action to goal) and the expectation of the human towards the robot's followed trajectory (goal to action). They proved that, on the one hand, trajectories are more legible if the probability to reach a certain goal given the current position is higher. On the other hand, trajectories are more predictable if their cost given in terms of the sum of squared velocities is lower. Moreover, it is shown that the optimisation of the legibility may lead to the generation of highly unpredictable trajectories and some unpredictability is often necessary to convey intent. A similar study [189] was aimed at optimising human visibility, reachability and ergonomics during handing-over tasks.

On the level of assembly cell design, it is demonstrated that the robot speed, the distance between the robot and the human, as well as the ability to communicate in advance the robot motion to the worker have an influence on the human propensity to accept the robot $[7,119,163,202,236]$. It is recognised that high robot speed, small distances and the lack of a signal communicating the start of the robot motion drastically reduce the human acceptance of the robot. Similarly, a properly designed HRC assembly cell can lead to about $6 \%$ time reduction toward assembly tasks completion, $20 \%$ more concurrent motion, and $3 \%$ less human idle time.

An important aspect affecting human propensity to work with a robot companion is the ability of the robot to dynamically change and adapt its operation in line with human behaviour and actions. According to the state-of-the-art, robot actions can be optimised on the basis of human behaviour $[75,180,194]$. A step forward in this direction is represented by a set of studies [81,181], showing that the success of a human-robot team also depends on the human level of adaptability in different situations. The robot should be able to interpret the setting and in particular, the level of human adaptability, and act properly.
Indeed, when the human can easily adapt, the robot can exploit human adaptability to guide the human towards a certain strategy. When the human is stubborn and she/he is not willing to comply, the robot should respect human preferences and optimise its choices considering the human preferences as a set of constraints. This leads to the development of the concept of mutual adaptation, where the human trust is considered during the definition of the robot strategy.

For a human-robot team to accomplish its goal, humans must trust that a robotic teammate will protect the interests and safety of every other individual in the team. The level of trust in the team is particularly critical in high-risk situations, such as in HRC assembly. Trust is important in these contexts because it directly affects the willingness of human to accept any robot-produced information, follow robots' suggestions, and thus benefit from the advantages inherent in an HRC system. In other words, trust affects much the decisions that humans make in uncertain or risky environments [74]. For example, the less an individual trusts a robot, the sooner he or she will intervene as it progresses toward task completion. In Fig. 43, Hancock et al. [74] summarised the factors that affect trust through a meta-analysis and classified them into three broad categories: human-related factors (including ability-based and human characteristic factors), robot-related factors (including performance- and attributebased factors), and environment-related factors (including team collaboration and task-based factors).

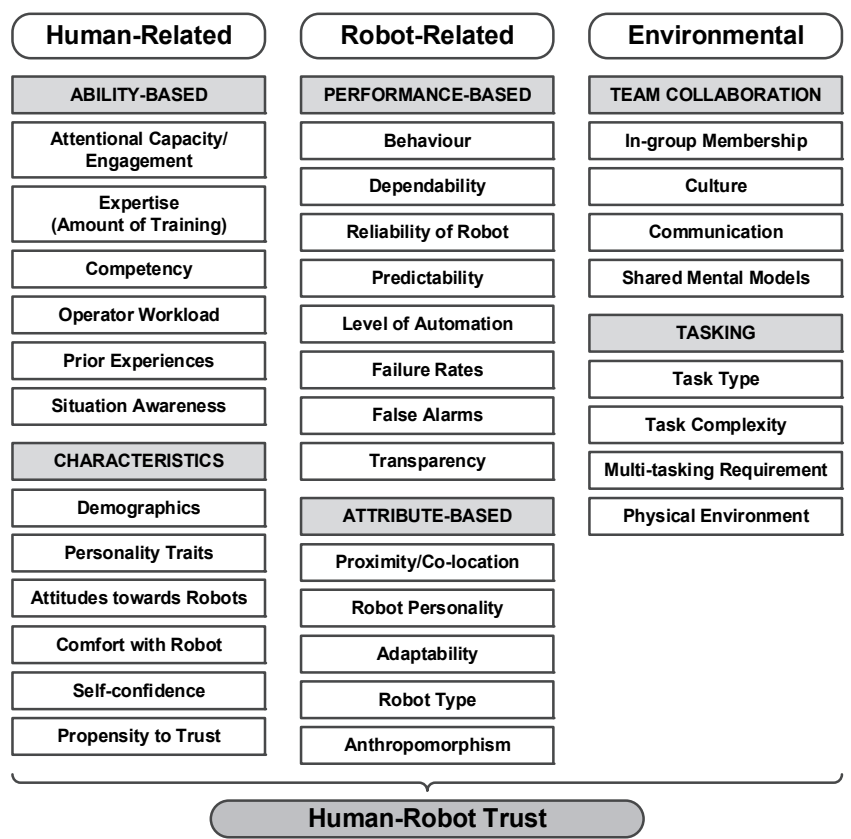

Fig. 43. Factors of trust development in HRC [74].

Finally, while social and emotional dimensions are apparently less subject to formal specifications, they, too, have been a focus of research in recent years. Jimenez and Dunkl [94] summarised the psychological aspects of work situations, where the authors pointed out the vital degrees of freedom (degree of autonomy, buffered resources, etc.) and comprehensible relations to the working and social environment (team alignment, goals, rewards, fairness, etc.) must lie within a preferable range. Regardless of cultural differences in consistent teamwork and management styles, the emotional alignment of the individual with the team has long since been recognised as vital both for performance and sustained mental health [165]. Various modelling approaches allow the mapping of the individual's attitude and group sentiment, both with surveys building a general corpus of conventions and customs within a given working culture, as well as with physiological measurement and machine learning techniques that respond to variations of the individual or deviations from past patterns and cultural average [165]. 


\section{Future research directions}

\subsection{Towards new industrial standards of HRC}

The potential of collaborative work of teams of multiple human and robotic agents creates such a new scenario of industrial production which requires not only novel scientific insights and technological solutions but also a new regulatory framework. ISO standards 10218-1 and -2 [84,87], amended with ISO/TS 15066 [85] define requirements for human-robot collaboration with an emphasis on safety (see Tables 2-4). Current standards for collaborative robotic environments already take an integrated approach with the compound of control, robot, end-effector, workpiece and task being simultaneously subject to assessment. This is a favourable starting point for further enhancement towards a complex multi-agent team. ISO/TS 15066 also distinguishes four modes of collaborative operation in a shared safeguarded area (safety-rated monitored stop, hand guiding, speed and separation monitoring, power and force limiting), taking the first step in removing rigid cell barriers of robotised workstations. These modes of operation will certainly determine possible ways of robots participating in collaborative teams that include humans. Nonetheless, ISO 10218 and ISO/TS 15066 deal with physical (mechanical) aspects only-mental and emotional issues remain largely unspecified.

Mental workload is though the subject of standards under ISO 10075 [88-90], which take it as pairs of (1) stimuli, originating in task requirements, physical conditions, as well as social, organisational, and societal factors, and (2) effect, resulting in mental strain reactions of the human (like activation and warming-up as facilitating effects, and mental fatigue, monotony, reduced vigilance and satiation as impairing effects). ISO 10075 clarifies that working environments should optimise mental stress to avoid both monotony and other forms of underload, as well as overload, fatigue and burnout. In this context, work equipment, ambient and social environment, and tasks are usually regarded as separate categories; however, it is expected that the introduction of social collaborative robots, and complex work environments that act as a team, will cross over the category limits, requiring a new integrated perspective in assessing, as well as designing such systems. While ISO 10075 does not specifically address such views, the perspectives of the standard are a consistent starting point for further synthesis. ISO 10075 largely remains on the level of the individual, and higherlevel perspectives are taken by the ISO 26000 on the "Guidance on social responsibility" [91]. These ethical guidelines are, however, too generic to be subjected to formal auditing or to provide firm specifications for human-robot teams working on the shop floor.

All in all, current standards do not yet address many key issues of mixed teams of humans and robots. Nevertheless, they outline some of the key aspects of a roadmap for such socially aware collaborative environments that set the baselines for a safe, anthropometric, mentally and socially satisfying environment for HRC. Intensive standardisation activities are ongoing and more realistic standards are anticipated.

\subsection{Modelling the human worker}

Continuous observation of behaviours and models of human disposition and emotion at workplace have been investigated intensively; however, industry-ripe application to work execution control at assembly workstations are still missing. There is a need to elaborate and populate models of human workforce and develop task execution control and HMC adaptation approaches that can establish individual worker preference profiles, pick up transient changes in the state of the individual worker, and tune both communication and acquired models accordingly.

\subsection{Digital twin for symbiotic HRC}

Designing, planning, operating, supervising and controlling assembly workcells where humans and robots collaborate requires accurate and almost in real-time updated digitalised models. However, here more is needed than a kind of replica of the devices, equipment, processes or even human operators. A socalled digital twin should combine and align all relevant aspects of modelling the function, structure and behaviour of the robotic cell including the worker, together with capturing the symbiotic interplay of the human and robotic agents. This includes representing the multimodal and bidirectional channels of communication and control as well. In the HRC context, the basis of the digital twin can be a linkage mechanism, which is capable of representing the geometric and kinematic relations of all static and moving objects in the workcell [80]. Time and again, the digital twin should be efficiently tuned - i.e. calibrated - to the real environment by using the actual measured data, so that both robot programming and operator instructions can automatically adapt to the actual situation.

\subsection{Optimising and adapting plans of redundant robots}

Redundancy-when the robot has more degrees of freedom than needed for performing a task-provides much opportunity for optimisation and adaptation, because, at least in principle, infinitely many joint configurations may result in executing the same task. However, when executing a set of tasks in a complex work environment which is characteristic to HRC assembly in particular, a number of technological and geometric constraints must be met. The solution requires a bi-directional transition between the task and configuration spaces for generating relevant and collision-free configurations only [56]. The computational complexity of such an approach is still too demanding for supporting real-time adaptive robot control.

\subsection{Shared tasks and team formation}

In today's industrial practice of assembly, collaborative workstations exist already. However, they do not exhibit all characteristics of a working environment hosting multiple collaborating humans and robotic agents. As discussed above, advanced methods of perception and sensor fusing, situation recognition, variable task assignment considering also skill levels, dynamic and adaptive planning, load balancing, as well as of human-robot mixed-initiative motion generation are known; however, it is still open how to harmonise autonomous decisions, change levels of autonomy and responsibility, and leave more abstract decisions to humans where conscious judgement is needed. In a multi-agent setting, the agents need to be aware of each other's states (and intentions), as well as the state of objects to successfully work together or safely coexist within the same physical space. For robots, a proper degree of awareness depends on the adequate implementation of communication or information acquisition channels and their introduction into their control. Being more autonomous but less predictable and reliable actors, humans need more support for being aware of the current situation. All these are prerequisites of true shared task execution and adaptive team formation in collaborative environments.

\subsection{Handling of exceptions, emergency and recovery}

Exceptions occur if the flow of processes involved is close or has passed the nominal limits; however, the processes can be brought back to normal without interruption, and the situation does not endanger the integrity or safety of human. Emergencies occur when danger of irrevocable damage is imminent. The latter case requires fast and guaranteed mitigation. Therefore, certain modes of communication do not apply to emergency signals. In addition, emergency mechanisms may have direct effect (e.g., emergency power-down by a single circuit breaker) that usually does not occur with communication in the usual sense.

\subsection{Work execution and training - learning by doing}

Current industrial practice does not explicitly foresee the 
integration of normal production operations with the handling of exceptions and emergencies and with recovery operations. Even though some methodologies address early fault prevention and detection, this is not necessarily equal to continuous feedback regarding operation results. Not only does this often result in the costly temporal and spatial separation of training from work execution, it also hampers orientation of the worker in ad-hoc work situations, e.g., in construction of individual products, or in the maintenance of poorly documented legacy equipment. Since training and quality check are typically separated from task execution, gradual skill development, live assistance and realtime feedback are obstructed. Industry-proof integrated process, robot and human models adequate in the context of HRC assembly must be elaborated where performance evaluation results (including those of quality checks) are fed back to support the improvement of the overall team. In any learning-by-doing scheme, the safety requirements must be continuously observed.

\subsection{Adaptive work instructions}

Human-robot interfaces, especially those conveying work instructions, often provide adaptability to the given worker in discrete steps, namely, by skill level categories. Adaptation to the worker's current (and changing) fitness for the current assembly task is, however, not part of industrial practice. The devices keeping track of the worker's awareness primarily serve safety purposes only. AR-based in-situ decision support to workers in dynamic HRC assembly environments deserves more attention to be both intuitive and mental stress-free. Work instructions needs to be adaptive to not only the changing competence level of individual workers but also the declining focus and concentration during the day or within the week.

\subsection{Programming-free robot control}

One potential application of data fusion in HRC assembly that should be actively explored is the multimodal fusion of human commands, for example, integration of both hand gestures and voice, to further improve the human command recognition rate and contribute to programming-free robot control.

Despite the increasing use of gestures and voice commands in HRC for robot control, they are less natural or practical in busy and noisy work environments. Instead of defined gestures and voice commands, recognition and prediction of human motions through deep learning provides better context awareness and less interruption to normal performance induced by signalling gestures. Haptic interaction with collaborating robots, especially for legacy industrial robots with few embedded sensors, is another way to achieve programming-free robot control. As an emerging research direction, brainwave-driven robot control can enhance symbiotic HRC assembly. 'Think ahead' combined with human motion prediction contributes to timely cognition and assistance from the robots, and adds value to the intelligence of an HRC system. Programming-free robot control can be facilitated by the use of event-driven function blocks with smart decision algorithms for detailed adaptive robot control, whereas the highlevel commands (e.g., gesture, voice, gaze, haptic, brainwave) serve as the triggering events to the function blocks for task execution. This unique combination paves the way towards the multimodal symbiotic HRC assembly.

\subsection{HRC as social activity with trust}

Although rarely applied in industrial production, research has already elaborated approaches for the modelling of emotional and social processes, as well as acquisition of individual or cultural profiles and their tuning to newly perceived changes. Existing results, therefore, provide a solid starting point for the elaboration of similar solutions for industrial applications in the HRC context. What is missing is the formation of mutual models of trust. When adding humans to shared robotic environments, this aspect is unavoidably important and deserves attention.

\subsection{Social responsibility of a new type of automation}

An HRC team forms a meta society where each team member bears certain social responsibilities for others such as safety and work ethics. The social stability of the team contributes to the level of automation for seamless HRC assembly. In such a new automation environment, mental stress and even psychological discomfort leading to any potential accident can be monitored and diagnosed via the brainwaves of human workers, which can be collected by sensors embedded in a safety helmet. Although an ethical issue, it contributes to safety assurance in workplaces.

\subsection{Limitations and challenges}

The wide interest of research and industry in the HRC related topics is proportional to the increased productivity and flexibility of production lines, as it combines human and robot capabilities [9,234]. However, the deployment of HRC in wide industrial applications is facing big challenges. Safety is the first concern. Although advanced methods/mechanisms have been developed in the past years, most of these methods are established in the lab environment. The hardware utilised are prototype-level devices that cannot be transferred to industry directly. More mature devices at higher Safety Integrity Level (SIL) are needed to further improve and exploit the research results. Moreover, the feasibility of the HRC solutions have been well evaluated, but the safety performance needs to be assessed systematically. For example, the stability, robustness, response time, redundant safety, backup solutions and emergency handling needs to be evaluated in a structured and standardised way.

As a result, the collision avoidance research need to impact the future standardisation as well. The current standards focus on distance and force/torque/energy limits to protect humans from severe injuries. However, there is no complete standard for the new technologies, e.g., vision-, ultrasonic-, depth-based sensing systems. The standards need to include those new technologies as a part of the robotic solutions.

Additionally, the social impacts of the collision avoidance need much attention as the operator needs to interact with the robot in an HRC setting without any wearable protections in daily work. The psychological reactions may change if the operator works with the robot for a long period. Sometimes, such changes may not be noticed by the operator himself/herself. Thus, more efforts as mentioned in Sections 8.10 and 8.11 are needed.

\section{Conclusions}

This paper presents the state-of-the-art of symbiotic humanrobot collaborative assembly. Research on HRC has been active for many years. Despite the advancement of HRC in recent years, confusions exist in different forms of human-robot relationships: coexistence, cooperation, interaction, and collaboration. There is a need to classify these relationships, and identify their unique features and characteristics with clear definitions. This paper is aimed to address these issues together with existing challenges and recent technological advancements. Within the context of symbiotic HRC assembly, topics covered include sensing and communication techniques, human safety assurance, dynamic assembly planning, programming-free robot control, and in-situ decision support to operators. 12 future research directions and challenges are also identified, hoping to shed some light on further advancement in the years to come. With the support of the latest technologies of sensing, communication, AI, AR and robot control, HRC will find its way to practical applications on shop floors in factories of the future.

\section{Acknowledgements}

The authors wish to thank Franz Dietrich, Zsolt Kemény and Stefania Pellegrinelli for their contributions to this keynote paper. This work is partially supported by EU FoF-06-2014 SYMBIO-TIC 
project (No. 637107) and Hungarian national grant No. GINOP2.3.2-15-2016-00002.

\section{References}

[1] Agravante DJ, Cherubini A, Kheddar A (2013) Using vision and haptic sensing for human-humanoid joint actions. IEEE Conf. Robot. Autom. Mechatronics, RAM - Proc. , pp 13-18.

[2] Ahmad R, Plapper P (2016) Safe and Automated Assembly Process using Vision Assisted Robot Manipulator. Procedia CIRP. Elsevier B.V., pp 771-776.

[3] Albu-Schaffer A, Hirzinger G (2002) Cartesian impedance control techniques for torque controlled light-weight robots. Robot. Autom. 2002. Proceedings. ICRA'02. IEEE Int. Conf. IEEE, pp 657-663.

[4] Alexopoulos K, Mavrikios D, Chryssolouris G (2013) ErgoToolkit: an ergonomic analysis tool in a virtual manufacturing environment. Int J Comput Integr Manuf 26(5):440-452.

[5] Andersen RH, Dalgaard L, Beck AB, Hallam J (2015) An architecture for efficient reuse in flexible production scenarios. Autom. Sci. Eng. (CASE), 2015 IEEE Int. Conf. IEEE, pp 151-157.

[6] Anton FD, Anton S, Borangiu T (2012) Human-Robot Natural Interaction and Collision Avoidance in Flexible Manufacturing Cells. IFAC Proc. Vol. IFAC, pp 835-840.

[7] Arai T, Kato R, Fujita M (2010) Assessment of operator stress induced by robot collaboration in assembly. CIRP Ann - Manuf Technol 59(1):5-8.

[8] Argall BD, Chernova S, Veloso M, Browning B (2009) A survey of robot learning from demonstration. Rob Auton Syst 57(5):469-483.

[9] Argyrou C, Giannoulis C, Sardelis A, Karagiannis P, Michalos G, Makris S (2018) A data fusion system for controlling the execution status in human-robot collaborative cells. Procedia CIRP 76 (CATS 2018) 7th CIPR Conf. Assem. Technol. Syst. Tianjin, China, China, pp 193-198.

[10] Asokan A, Pothen AJ, Vijayaraj RK (2016) ARMatron-A Wearable Gesture Recognition Glove: for Control of Robotic Devices in Disaster Management and
Human Rehabilitation. Robot. Autom. Humanit. Appl. (RAHA), 2016 Int. Conf. IEEE, pp 1-5.

[11] Augustsson S, Christiernin LG, Bolmsjö G (2014) Human and robot interaction based on safety zones in a shared work environment. Proc. 2014 ACM/IEEE Int. Conf. Human-robot Interact. pp 118-119.

[12] Augustsson S, Olsson J, Christiernin LG, Bolmsjö G (2014) How to transfer information between collaborating human operators and industrial robots in an assembly. Nord. Conf. Human-Computer Interact. , pp 286-294.

[13] Azuma RT (1997) A survey of augmented reality. Presence Teleoperators Virtual Environ 6(4):355-385.

[14] Baccouche M, Mamalet F, Wolf C, Garcia C, Baskurt A (2011) Sequential deep learning for human action recognition. Int. Work. Hum. Behav. Underst. Springer, pp 29-39.

[15] Bänziger T, Kunz A, Wegener K (2018) Optimizing human-robot task allocation using a simulation tool based on standardized work descriptions. J Intell Manuf $1-14$

[16] Barreiro J, Boyce M, Do M, Frank J, Iatauro M, Kichkaylo T, Morris P, Ong J, Remolina E, Smith T (2012) EUROPA: a platform for AI planning, scheduling, constraint programming, and optimization. 4th Int. Compet. Knowl. Eng. Plan. Sched.

[17] Bdiwi M, Pfeifer M, Sterzing A (2017) A new strategy for ensuring human safety during various levels of interaction with industrial robots. CIRP Ann Manuf Technol 66(1):453-456.

[18] Berg J, Reinhart G (2017) An Integrated Planning and Programming System for Human-Robot-Cooperation. Procedia CIRP 63:95-100.

[19] Berman S, Stern H (2012) Sensors for gesture recognition systems. IEEE Trans Syst Man, Cybern Part C (Applications Rev 42(3):277-290.

[20] Berri R, Wolf D, Osório F (2014) Telepresence Robot with Image-Based Face Tracking and 3D Perception with Human Gesture Interface Using Kinect Sensor. Robot. SBR-LARS Robot. Symp. Rob. (SBR LARS Rob. 2014 Jt. Conf. IEEE, pp 205-210.

[21] Bi ZM, Wang L (2010) Advances in 3D data acquisition and processing for industrial applications. Robot Comput Integr Manuf 26(5):403-413.

[22] Bi ZM, Lang SYT, Wang L (2008) Improved control and simulation models of a tricycle collaborative robot. J Intell Manuf 19(6):715-722.

[23] Bi ZM, Wang L (2010) Dynamic control model of a cobot with three omniwheels. Robot Comput Integr Manuf 26(6):558-563.

[24] Bi ZM, Wang L, Lang SYT (2007) Current status of reconfigurable assembly systems. Int J Manuf Res 2(3):303-328.

[25] Bicchi A, Peshkin MA, Colgate JE (2008) Safety for Physical Human-Robot Interaction. Springer Handb. Robot. , pp 1335-1348.

[26] Bischoff R, Kurth J, Schreiber G, Koeppe R, Albu-Schäffer A, Beyer A, Eiberger O, Haddadin S, Stemmer A, Grunwald G (2010) The KUKA-DLR Lightweight Robot arm-a new reference platform for robotics research and manufacturing. Robot. (ISR), 2010 41st Int. Symp. 2010 6th Ger. Conf. Robot. VDE, pp 1-8.

[27] Bobka P, Germann T, Heyn JK, Gerbers R, Dietrich F, Dröder K (2016) Simulation Platform to Investigate Safe Operation of Human-Robot Collaboration Systems. Procedia CIRP., pp 187-192.

[28] Bruno G, Antonelli D (2018) Dynamic task classification and assignment for the management of human-robot collaborative teams in workcells. Int J Adv Manuf Technol 98(9-12):2415-2427.

[29] Bruyninckx H, Klotzbücher M, Hochgeschwender N, Kraetzschmar G, Gherardi L, Brugali D (2013) The BRICS component model: a model-based development paradigm for complex robotics software systems. Proc. 28th Annu. ACM Symp. Appl. Comput. ACM, pp 1758-1764.

[30] Calinon S, Sardellitti I, Caldwell DG (2010) Learning-based control strategy for safe human-robot interaction exploiting task and robot redundancies. Intell. Robot. Syst. (IROS), 2010 IEEE/RSJ Int. Conf. IEEE, pp 249-254.

[31] Canedo-Rodríguez A, Alvarez-Santos V, Regueiro C V, Iglesias R, Barro S, Presedo J (2016) Particle filter robot localisation through robust fusion of laser, WiFi, compass, and a network of external cameras. Inf Fusion 27:170188.

[32] Cantelli L, Muscato G, Nunnari M, Spina D (2015) A joint-angle estimation method for industrial manipulators using inertial sensors. IEEE/ASME Trans Mechatronics 20(5):2486-2495.

[33] Carlson J, Murphy RR (2005) How UGVs physically fail in the field. IEEE Trans Robot 21(3):423-437.

[34] Casalino A, Guzman S, Zanchettin AM, Rocco P (2018) Human pose estimation in presence of occlusion using depth camera sensors, in human-robot coexistence scenarios. 2018 IEEE/RSJ Int. Conf. Intell. Robot. Syst. IEEE, pp 17 .

[35] Casalino A, Messeri C, Pozzi M, Zanchettin AM, Rocco P, Prattichizzo D (2018) Operator Awareness in Human-Robot Collaboration Through Wearable Vibrotactile Feedback. IEEE Robot Autom Lett 3(4):4289-4296.

[36] Cha Y, Seo J, Kim J-S, Park J-M (2017) Human-computer interface glove using flexible piezoelectric sensors. Smart Mater Struct 26(5):57002.

[37] Chen F, Sekiyama K, Huang J, Sun B, Sasaki H, Fukuda T (2011) An assembly strategy scheduling method for human and robot coordinated cell manufacturing. Int J Intell Comput Cybern 4(4):487-510.

[38] Chen M, Nikolaidis S, Soh H, Hsu D, Srinivasa S (2018) Planning with trust for human-robot collaboration. Proc. 2018 ACM/IEEE Int. Conf. Human-Robot Interact. ACM, pp 307-315.

[39] Cherubini A, Passama R, Crosnier A, Lasnier A, Fraisse P (2016) Collaborative manufacturing with physical human-robot interaction. Robot Comput Integr Manuf 40:1-13.

[40] Cherubini A, Passama R, Fraisse P, Crosnier A (2015) A unified multimodal control framework for human-robot interaction. Rob Auton Syst 70:106-115.

[41] Chong JWS, Nee AYC, Youcef-Toumi K, Ong SK (2005) An application of augmented reality (ar) in the teaching of an arc welding robot.

[42] Chong JWS, Ong SK, Nee AYC, Youcef-Youmi K (2009) Robot programming using augmented reality: An interactive method for planning collision-free paths. Robot Comput Integr Manuf 25(3):689-701.

[43] Cirillo A, Ficuciello F, Natale C, Pirozzi S, Villani L (2016) A Conformable Force/Tactile Skin for Physical Human-Robot Interaction. IEEE Robot Autom Lett 1(1):41-48.

[44] Colombo A, Fontanelli D, Macii D, Palopoli L (2014) Flexible indoor localization and tracking based on a wearable platform and sensor data fusion. IEEE Trans Instrum Meas 63(4):864-876.

[45] Corrales JA, Candelas FA, Torres F (2011) Safe human-robot interaction based on dynamic sphere-swept line bounding volumes. Robot Comput Integr Manuf 27(1):177-185.

[46] Dániel B, Korondi P, Thomessen T (2012) Joint level collision avoidance for industrial robots. IFAC Proc Vol 45(22):655-658.

[47] Denkena B, Wörn H, Apitz R, Bischoff R, Hein B, Kowalski P, Mages D, Schuler H (2005) Roboterprogrammierung in der Fertigung. Springer 9:656-660.

[48] Dombrowski U, Stefanak T, Reimer A (2018) Simulation of human-robot collaboration by means of power and force limiting: Paper ID: 1156. Procedia Manuf 17:134-141.

[49] Dominick V, Markus K, Herman B (2014) The 5C-based architectural Composition Pattern: lessons learned from re-developing the iTaSC framework for constraint-based robot programming. J Softw Eng Robot 5(1):17-35.

[50] Dong W, Xiao L, Hu W, Zhu C, Huang Y, Yin Z (2017) Wearable human-machine interface based on PVDF piezoelectric sensor. Trans Inst Meas Control 39(4):398-403

[51] Dragan A Srinivasa S (2014) Integrating human observer inferences into robot motion planning. Auton Robots 37(4):351-368.

[52] Durrant-Whyte H, Bailey T (2006) Simultaneous localization and mapping: part I. IEEE Robot Autom Mag 13(2):99-110.

[53] Dvorák F, Barták R, Bit-Monnot A, Ingrand F, Ghallab M (2014) Planning and acting with temporal and hierarchical decomposition models. Tools with Artif. Intell. (ICTAI), 2014 IEEE 26th Int. Conf. IEEE, pp 115-121.

[54] Ebert D, Komuro T, Namiki A, Ishikawa M (2005) Safe human-robotcoexistence: Emergency-stop using a high-speed vision-chip. 2005 IEEE/RS] Int. Conf. Intell. Robot. Syst. IROS. , pp 1821-1826.

[55] Ebert DM, Henrich DD (2002) Safe human-robot-cooperation: Image-based collision detection for industrial robots. Intell. Robot. Syst. 2002. IEEE/RSJ Int. Conf. IEEE, pp 1826-1831.

[56] Erdős G, Kovács A, Váncza J (2016) Optimized joint motion planning for redundant industrial robots. CIRP Ann - Manuf Technol 65(1):451-454.

[57] Farrell RG, Lenchner J, Kephjart JO, Webb AM, Muller MiJ, Erikson TD, Melville DO, Bellamy RKE, Gruen DM, Connell JH (2016) Symbiotic cognitive computing. AI Mag 37(3):81-93.

[58] Fischer M, Henrich D (2009) 3D collision detection for industrial robots and unknown obstacles using multiple depth images. Springer, Berlin, Heidelberg

[59] Flacco F, Kroeger T, De Luca A, Khatib O (2015) A Depth Space Approach for Evaluating Distance to Objects: with Application to Human-Robot Collision Avoidance. J Intell Robot Syst Theory Appl 80:7-22.

[60] Flacco F, Kröger T, De Luca A, Khatib 0 (2012) A depth space approach to human-robot collision avoidance. Robot. Autom. (ICRA), 2012 IEEE Int. Conf. IEEE, pp 338-345

[61] Garć́a JG, Robertsson A, Ortega JG, Johansson R (2008) Sensor fusion for compliant robot motion control. IEEE Trans Robot 24(2):430-441.

[62] de Gea Fernández J, Mronga D, Günther M, Knobloch T, Wirkus M, Schröer M, Trampler M, Stiene S, Kirchner E, Bargsten V (2017) Multimodal sensor-based whole-body control for human-robot collaboration in industrial settings. Rob Auton Syst 94:102-119.

[63] Gecks T, Henrich D (2005) Human-robot cooperation: Safe pick-and-place operations. Proc. - IEEE Int. Work. Robot Hum. Interact. Commun. , pp 549554.

[64] Geravand M, Flacco F, De Luca A (2013) Human-robot physical interaction and collaboration using an industrial robot with a closed control architecture. 2013 IEEE Int. Conf. Robot. Autom. , pp 4000-4007. 
[65] Gerevini AE, Haslum P, Long D, Saetti A, Dimopoulos Y (2009) Deterministic planning in the fifth international planning competition: PDDL3 and experimental evaluation of the planners. Artif Intell 173(5-6):619-668.

[66] Ghallab M, Laruelle H (1994) Representation and Control in IxTeT, a Temporal Planner. AIPS'94 Proc. Second Int. Conf. Artif. Intell. Plan. Syst. , pp 61-67.

[67] Gkournelos C, Karagiannis P, Kousi N, Michalos G, Koukas S, Makris S (2018) Application of Wearable Devices for Supporting Operators in Human-Robot Cooperative Assembly Tasks. (CATS 2018) 7th CIPR Conf. Assem. Technol. Syst. Tianjin, China, China, pp 177-182.

[68] Grunwald G, Schreiber G, Albu-Schaffer A, Hirzinger G (2001) Touch: The direct type of human interaction with a redundant service robot. Robot Hum. Interact. Commun. 2001. Proceedings. 10th IEEE Int. Work. IEEE, pp 347-352.

[69] Haddadin S, Albu-Schäffer A, Hirzinger G (2009) Requirements for Safe Robots: Measurements, Analysis and New Insights. Int J Rob Res 28(1112):1507-1527.

[70] Haddadin S, Khoury A, Rokahr T, Parusel S, Burgkart R, Bicchi A, Albu-Schäffer A (2012) On making robots understand safety: Embedding injury knowledge into control. Int J Rob Res 31(13):1578-1602.

[71] Haddadin S, Albu-Schäffer A, Hirzinger G (2007) Safety Evaluation of Physical Human-Robot Interaction via Crash-Testing. Proc. Robot. Sci. Syst. , pp $217-$ 224.

[72] Haddadin S, Albu-Schäffer A, De Luca A, Hirzinger G (2008) Evaluation of collision detection and reaction for a human-friendly robot on biological tissues. Proc IARP 2008 1-7.

[73] Haddadin S, Albu-Schäffer A, De Luca A, Hirzinger G (2008) Collision detection and reaction: A contribution to safe physical human-robot interaction. 2008 IEEE/RSJ Int. Conf. Intell. Robot. Syst. , pp 3356-3363.

[74] Hancock PA, Billings DR, Schaefer KE, Chen JYC, de Visser EJ, Parasuraman R (2011) A Meta-Analysis of Factors Affecting Trust in Human-Robot Interaction. Hum Factors J Hum Factors Ergon Soc 53(5):517-527.

[75] Hawkins KP, Vo N, Bansal S, Bobick AF (2013) Probabilistic human action prediction and wait-sensitive planning for responsive human-robot collaboration. Humanoid Robot. (Humanoids), 2013 13th IEEE-RAS Int. Conf. IEEE, pp 499-506.

[76] Hayes B, Scassellati B (2015) Effective robot teammate behaviors for supporting sequential manipulation tasks. Intell. Robot. Syst. (IROS), 2015 IEEE/RSJ Int. Conf. IEEE, pp 6374-6380.

[77] Heinzmann J, Zelinsky A (2003) Quantitative Safety Guarantees for Physical Human-Robot Interaction. Int J Rob Res 22(7-8):479-504.

[78] Héliot R, Espiau B (2008) Multisensor Input for CPG-Based Sensory---Motor Coordination. IEEE Trans Robot 24(1):191-195

[79] Hong S, Lee H, Lee J, Kwon J, Han S, Suh YD, Cho H, Shin J, Yeo J, Ko SH (2015) Highly stretchable and transparent metal nanowire heater for wearable electronics applications. Adv Mater 27(32):4744-4751.

[80] Horváth G, Erdős G (2017) Point cloud based robot cell calibration. CIRP Ann 66(1):145-148

[81] Huang C-M, Cakmak M, Mutlu B (2015) Adaptive Coordination Strategies for Human-Robot Handovers. Proc. 2015 Robot. Sci. Syst. Conf.

[82] Huang S, Ishikawa M, Yamakawa Y (2018) An Active Assistant Robotic System Based on High-Speed Vision and Haptic Feedback for Human-Robot Collaboration. IECON 2018-44th Annu. Conf. IEEE Ind. Electron. Soc. IEEE, pp 3649-3654.

[83] IEC (2005) IEC 61499. Function blocks for industrial-process measurement and control systems- part 2: Software Tools Requirements. IEC, Geneva

[84] ISO (2011) ISO 10218-1: Robots and robotic devices - Safety requirements for industrial robots - Part 1: Robots.

[85] ISO (2016) ISO/TS 15066 Robotics and robotic devices - Collaborative robots.

[86] ISO (2010) ISO 13855 Safety of machinery -- Positioning of safeguards with respect to the approach speeds of parts of the human body.

[87] ISO (2011) ISO 10218-2: Robots and robotic devices - Safety requirements for industrial robots - Part 2: Robot systems and integration.

[88] ISO (2017) ISO 10075-1: Ergonomic principles related to mental workload -Part 1: General issues and concepts, terms and definitions.

[89] ISO (1996) ISO 10075-2: Ergonomic principles related to mental workload -Part 2: Design principles.

[90] ISO (2004) ISO 10075-3: Ergonomic principles related to mental workload -Part 3: Principles and requirements concerning methods for measuring and assessing mental workload.

[91] ISO (2010) ISO 26000: Guidance on social responsibility.

[92] Javdani S, Srinivasa SS, Bagnell JA (2015) Shared autonomy via hindsight optimization. arXiv Prepr. arXiv1503.07619

[93] Ji W, Yin S, Wang L (2018) A Virtual Training Based Programming-Free Automatic Assembly Approach for Future Industry. IEEE Access 6:4386543873.

[94] Jimenez P, Dunkl A (2017) The Buffering Effect of Workplace Resources on the Relationship between the Areas of Worklife and Burnout. Front. Psychol. 8:

[95] Johannsmeier L, Haddadin S (2017) A hierarchical human-robot interactionplanning framework for task allocation in collaborative industrial assembly processes. IEEE Robot Autom Lett 2(1):41-48.

[96] Kaiser L, Schlotzhauer A, Brandstötter M (2018) Safety-Related Risks and Opportunities of Key Design-Aspects for Industrial Human-Robot Collaboration. Int. Conf. Interact. Collab. Robot. Springer, pp 95-104.

[97] Kappassov Z, Corrales J-A, Perdereau V (2015) Tactile sensing in dexterous robot hands. Rob Auton Syst 74:195-220.

[98] Karami A-B, Jeanpierre L, Mouaddib A-I (2010) Human-robot collaboration for a shared mission. Proc. 5th ACM/IEEE Int. Conf. Human-robot Interact. IEEE Press, pp 155-156.

[99] Kardos C, Kemény Z, Kovács A, Pataki B, Váncza J (2018) Context-dependent multimodal communication in human-robot collaboration. PROCEDIA CIRP 72:15-20.

[100] Kardos C, Kovács A, Váncza J (2017) Decomposition approach to optimal feature-based assembly planning. CIRP Ann - Manuf Technol 66(1):417-420.
[101] Kardos C, Váncza J (2018) Mixed-initiative assembly planning combining geometric reasoning and constrained optimization. CIRP Ann - Manuf Technol 67(1):463-466.

[102] Khaleghi B, Khamis A, Karray FO, Razavi SN (2013) Multisensor data fusion: A review of the state-of-the-art. Inf Fusion 14(1):28-44.

[103] Knoop S, Vacek S, Dillmann R (2009) Fusion of 2D and 3D sensor data for articulated body tracking. Rob Auton Syst 57(3):321-329.

[104] Koch H, Konig A, Weigl-Seitz A, Kleinmann K, Suchy J (2013) Multisensor contour following with vision, force, and acceleration sensors for an industrial robot. IEEE Trans Instrum Meas 62(2):268-280.

[105] Kokkalis K, Michalos G, Aivaliotis P, Makris S (2018) An approach for implementing power and force limiting in sensorless industrial robots. (CATS 2018) 7th CIPR Conf. Assem. Technol. Syst. Tianjin, China, China, pp 138-143.

[106] Kortenkamp D, Simmons R, Brugali D (2016) Robotic systems architectures and programming. Springer Handb. Robot. Springer, pp 283-306.

[107] Kousi N, Michalos G, Aivaliotis S, Makris S (2018) An outlook on future assembly systems introducing robotic mobile dual arm workers. Procedia CIRP 72:33-38.

[108] Krüger J, Nickolay B, Heyer P, Seliger G (2005) Image based 3D surveillance for flexible man-robot-cooperation. CIRP Ann - Manuf Technol 54(1):19-22.

[109] Krüger J, Surdilovic D (2008) Robust control of force-coupled human-robotinteraction in assembly processes. CIRP Ann - Manuf Technol 57(1):41-44.

[110] Krüger J, Wang L, Verl A, Bauernhansl T, Carpanzano E, Makris S, Fleischer J, Reinhart G, Franke J, Pellegrinelli S (2017) Innovative control of assembly systems and lines. CIRP Ann - Manuf Technol 66(2):707-730.

[111] Krüger J, Lien TKTK, Verl A (2009) Cooperation of human and machines in assembly lines. CIRP Ann - Manuf Technol 58(2):628-646.

[112] Krüger J, Nickolay B, Heyer P, Seliger G (2005) Image based 3D surveillance for flexible man-robot-cooperation. CIRP Ann - Manuf Technol 54(1):19-22.

[113] Kulíc D, Croft E (2007) Affective state estimation for human-robot interaction. IEEE Trans Robot 23(5):991-1000.

[114] Kulić D, Croft E (2007) Pre-collision safety strategies for human-robot interaction. Auton Robots 22(2):149-164

[115] Kulić D, Croft EA (2006) Real-time safety for human-robot interaction. Rob Auton Syst 54(1):1-12.

[116] Laffranchi M, Tsagarakis NG, Caldwell DG (2009) Safe human robot interaction via energy regulation control. IEEE/RSJ Int. Conf. Intell. Robot. Syst. , pp 35-41.

[117] Lambrecht J, Kleinsorge M, Krüger J (2011) Markerless gesture-based motion control and programming of industrial robots. Emerg. Technol. Fact. Autom. (ETFA), 2011 IEEE 16th Conf. IEEE, pp 1-4.

[118] Lambrecht J, Krüger J (2012) Spatial programming for industrial robots based on gestures and augmented reality. Intell. Robot. Syst. (IROS), 2012 IEEE/RSJ Int. Conf. IEEE, pp 466-472.

[119] Lasota PA, Shah JA (2015) Analyzing the effects of human-aware motion planning on close-proximity human-robot collaboration. Hum Factors 57(1):21-33.

[120] Lasota P, Nikolaidis S, Shah JA (2013) Developing an adaptive robotic assistant for close proximity human-robot collaboration in space. AIAA Infotech@ Aerosp. (I@ A) Conf. , p 4806.

[121] Lee JY, Rhee G (2008) Context-aware 3D visualization and collaboration services for ubiquitous cars using augmented reality. Int J Adv Manuf Technol 37(5-6):431-442.

[122] Lemai S, Ingrand F (2004) Interleaving temporal planning and execution in robotics domains. AAAI. , pp 617-622.

[123] Levine S, Pastor P, Krizhevsky A, Quillen D (2016) Learning hand-eye coordination for robotic grasping with large-scale data collection. Int. Symp. Exp. Robot. Springer, pp 173-184.

[124] Li T, Zou J, Xing F, Zhang M, Cao X, Wang N, Wang ZL (2017) From dual-mode triboelectric nanogenerator to smart tactile sensor: a multiplexing design. ACS Nano 11(4):3950-3956.

[125] Li X, Gao L, Zhang C, Shao X (2010) A review on integrated process planning and scheduling. Int J Manuf Res 5(2):161-180.

[126] Licklider JCR (1960) Man-computer symbiosis. IRE Trans Hum factors Electron (1):4-11.

[127] Lin MC (1993) Efficient Collision Detection for Animation and Robotics. Citeseer

[128] Lindsay A, Read J, Ferreira JF, Hayton T, Porteous J, Gregory PJ (2017) Framer: Planning models from natural language action descriptions.

[129] Liu B, Zhang F, Qu X (2015) A method for improving the pose accuracy of a robot manipulator based on multi-sensor combined measurement and data fusion. Sensors 15(4):7933-7952.

[130] Liu H, Fang T, Zhou T, Wang L (2018) Towards Robust Human-Robot Collaborative Manufacturing: Multimodal Fusion. IEEE Access 6:74762-74771.

[131] Liu H, Fang T, Zhou T, Wang Y, Wang L (2018) Deep learning-based multimodal control interface for human-robot collaboration. Procedia CIRP 72(1):3-8.

[132] Liu H, Wang L (2017) Gesture recognition for human-robot collaboration: A review. Int J Ind Ergon 1-13.

[133] Liu H, Wang L (2017) Human motion prediction for human-robot collaboration. J Manuf Syst 1-8.

[134] Liu H, Wang L (2017) An AR-based Worker Support System for Human-Robot Collaboration. Procedia Manuf., pp 1-9.

[135] Liu H, Wang Y, Ji W, Wang L (2018) A Context-Aware Safety System for Human-Robot Collaboration. Procedia Manuf 17:238-245.

[136] Liu S, Wu X, Zhang D, Guo C, Wang P, Hu W, Li X, Zhou X, Xu H, Luo C (2017) Ultrafast dynamic pressure sensors based on graphene hybrid structure. ACS Appl Mater Interfaces 9(28):24148-24154.

[137] Loughlin C, Albu-Schäffer A, Haddadin S, Ott C, Stemmer A, Wimböck T, Hirzinger G (2007) The DLR lightweight robot: design and control concepts for robots in human environments. Ind Robot an Int J 34(5):376-385.

[138] Lu S, Chung JH, Velinsky SA (2005) Human-robot collision detection and identification based on wrist and base force/torque sensors. Robot. Autom. 2005. ICRA 2005. Proc. 2005 IEEE Int. Conf. IEEE, pp 3796-3801. 
[139] De Luca A, Albu-Schaffer A, Haddadin S, Hirzinger G (2006) Collision detection and safe reaction with the DLR-III lightweight manipulator arm. Intell. Robot Syst. 2006 IEEE/RSJ Int. Conf. IEEE, pp 1623-1630.

[140] De Luca A, Ferrajoli L (2008) Exploiting robot redundancy in collision detection and reaction. Intell. Robot. Syst. 2008. IROS 2008. IEEE/RSJ Int. Conf. IEEE, pp 3299-3305.

[141] De Luca A, Flacco F (2012) Integrated control for pHRI: Collision avoidance, detection, reaction and collaboration. Proc. IEEE Int. Conf. Biomed. Robot. Biomechatronics. , pp 288-295.

[142] De Luca A, Flacco F, Bicchi A, Schiavi R (2009) Nonlinear decoupled motionstiffness control and collision detection/reaction for the VSA-II variable stiffness device. Intell. Robot. Syst. 2009. IROS 2009. IEEE/RSJ Int. Conf. IEEE, pp 5487-5494.

[143] De Luca A, Mattone R (2005) Sensorless robot collision detection and hybrid force/motion control. Robot. Autom. 2005. ICRA 2005. Proc. 2005 IEEE Int. Conf. IEEE, pp 999-1004.

[144] Maeda G, Ewerton M, Neumann G, Lioutikov R, Peters J (2017) Phase estimation for fast action recognition and trajectory generation in humanrobot collaboration. Int J Rob Res 1-16.

[145] Maeda G, Ewerton M, Neumann G, Lioutikov R, Peters J (2017) Phase estimation for fast action recognition and trajectory generation in humanrobot collaboration. Int J Rob Res 1-16.

[146] Mainprice J, Berenson D (2013) Human-robot collaborative manipulation planning using early prediction of human motion. Intell. Robot. Syst. (IROS), 2013 IEEE/RSJ Int. Conf. IEEE, pp 299-306.

[147] Mainprice J, Sisbot EA, Jaillet L, Cortés J, Alami R, Siméon T (2011) Planning human-aware motions using a sampling-based costmap planner. Robot. Autom. (ICRA), 2011 IEEE Int. Conf. IEEE, pp 5012-5017.

[148] Makris S, Michalos G, Chryssolouris G (2011) A Pushlet-Based Wireless Information Environment for Mobile Operators in Human Based Assembly Lines. 7th Int. Conf. Digit. Enterp. Technol. (DET 2011), Athens, Greece. , pp 622-631.

[149] Makris S, Karagiannis P, Koukas S, Matthaiakis A-S (2016) Augmented reality system for operator support in human-robot collaborative assembly. CIRP Ann - Manuf Technol 65(1):61-64.

[150] Makris S, Pintzos G, Rentzos L, Chryssolouris G (2013) Assembly support using AR technology based on automatic sequence generation. CIRP Ann - Manuf Technol 62(1):9-12.

[151] Makris S, Tsarouchi P, Matthaiakis A-S, Athanasatos A, Chatzigeorgiou X, Stefos M, Giavridis K, Aivaliotis S (2017) Dual arm robot in cooperation with humans for flexible assembly. CIRP Ann - Manuf Technol 66(1):13-16

[152] Makris S, Tsarouchi P, Surdilovic D, Krüger J (2014) Intuitive dual arm robot programming for assembly operations. CIRP Ann - Manuf Technol 63(1):13-16.

[153] Martinelli A (2012) Vision and IMU data fusion: Closed-form solutions for attitude, speed, absolute scale, and bias determination. IEEE Trans Robot 28(1):44-60.

[154] Mayer MC, Orlandini A, Umbrico A (2016) Planning and execution with flexible timelines: a formal account. Acta Inform 53(6-8):649-680.

[155] McDermott D, Ghallab M, Howe A, Knoblock C, Ram A, Veloso M, Weld D, Wilkins D (1998) PDDL-the planning domain definition language.

[156] McGhan CLR, Nasir A, Atkins EM (2015) Human intent prediction using Markov decision processes. J Aerosp Inf Syst 12(5):393-397.

[157] McPherson DL, Scobee DRR, Menke J, Yang AY, Sastry SS (2018) Modeling Supervisor Safe Sets for Improving Collaboration in Human-Robot Teams. 2018 IEEE/RSJ Int. Conf. Intell. Robot. Syst. IEEE, pp 861-868.

[158] Meguenani A, Padois V, Da Silva J, Hoarau A, Bidaud P (2016) Energy based control for safe human-robot physical interaction. Int. Symp. Exp. Robot. Springer, pp 809-818.

[159] Michalos G, Karagiannis P, Makris S, Tokçalar Ö, Chryssolouris G (2016) Augmented reality (AR) applications for supporting human-robot interactive cooperation. Procedia CIRP 41:370-375.

[160] Michalos G, Kousi N, Karagiannis P, Gkournelos C, Dimoulas K, Koukas S, Mparis K, Papavasileiou A, Makris S (2018) Seamless human robot collaborative assembly-An automotive case study. Mechatronics (55):194211

[161] Michalos G, Makris S, Rentzos L, Chryssolouris G (2010) Dynamic job rotation for workload balancing in human based assembly systems. CIRP J Manuf Sci Technol 2(3):153-160.

[162] Michalos G, Makris S, Spiliotopoulos J, Misios I, Tsarouchi P, Chryssolouris G (2014) ROBO-PARTNER: Seamless Human-Robot cooperation for intelligent, flexible and safe operations in the assembly factories of the future. Procedia CIRP 23:71-76.

[163] Michalos G, Makris S, Tsarouchi P, Guasch T, Kontovrakis D, Chryssolouris G (2015) Design considerations for safe human-robot collaborative workplaces. Procedia CIRP 37:248-253.

[164] Milgram P, Colquhoun H (1999) A taxonomy of real and virtual world display integration. Springer-Verlag, Berlin Heidelberg

[165] Moerland TM, Broekens J, Jonker CM (2017) Emotion in reinforcement learning agents and robots: a survey. Mach Learn (5):1-38.

[166] Mohammed A, Schmidt B, Wang L (2017) Active Collision Avoidance for Human-Robot Collaboration Driven by Vision Sensors. Int J Comput Integr Manuf 30(9):970-980.

[167] Mohammed A, Schmidt B, Wang L (2017) Energy-efficient robot configuration for assembly. J Manuf Sci Eng 139(5):51007.

[168] Mohammed A, Wang L (2018) Brainwaves driven human-robot collaborative assembly. CIRP Ann - Manuf Technol 67(1):13-16.

[169] Monje CA, Pierro P, Balaguer C (2011) A new approach on human-robot collaboration with humanoid robot RH-2. Robotica 29(6):949-957.

[170] Monostori L, Váncza J, Kumara SRT (2006) Agent-Based Systems for Manufacturing. CIRP Ann-Manuf Technol 55(2):697-720.

[171] Monostori L, Kádár B, Bauernhansl T, Kondoh S, Kumara S, Reinhart G, Sauer O, Schuh G, Sihn W, Ueda K (2016) Cyber-physical systems in manufacturing. CIRP Ann - Manuf Technol 65(2):621-641.
[172] Morato C, Kaipa KN, Zhao B, Gupta SK (2014) Toward safe human robot collaboration by using multiple kinects based real-time human tracking. J Comput Inf Sci Eng 14(1):11006.

[173] Morinaga S, Kosuge K (2003) Collision detection system for manipulator based on adaptive impedance control law. Robot. Autom. 2003. Proceedings. ICRA'03. IEEE Int. Conf. IEEE, pp 1080-1085.

[174] Morris P (2014) Dynamic controllability and dispatchability relationships. Int. Conf. AI OR Tech. Constriant Program. Comb. Optim. Probl. Springer, pp 464479.

[175] Müller R, Peifer R, Mailahn O (2018) Objectification of Assembly Planning for the Implementation of Human-Robot Cooperation. Int. Conf. Appl. Hum. Factors Ergon. Springer, pp 24-34.

[176] Muscettola N (1993) HSTS: Integrating planning and scheduling. CARNEGIEMELLON UNIV PITTSBURGH PA ROBOTICS INST

[177] Musić S, Hirche S (2017) Control sharing in human-robot team interaction Annu Rev Control 44:342-354.

[178] Nee AYC, Ong SK, Chryssolouris G, Mourtzis D (2012) Augmented reality applications in design and manufacturing. CIRP Ann - Manuf Technol 61(2):657-679.

[179] Neto P, Norberto Pires J, Paulo Moreira A (2010) High-level programming and control for industrial robotics: using a hand-held accelerometer-based input device for gesture and posture recognition. Ind Robot An Int J 37(2):137-147.

[180] Nikolaidis S, Shah J (2012) Human-robot interactive planning using crosstraining: A human team training approach. Infotech@ Aerosp. 2012. , p 2536.

[181] Nikolaidis S, Zhu YX, Hsu D, Srinivasa S (2017) Human-robot mutual adaptation in shared autonomy. Proc. 2017 ACM/IEEE Int. Conf. Human-Robot Interact. ACM, pp 294-302.

[182] Nikolakis N, Maratos V, Makris S (2019) A cyber physical system (CPS) approach for safe human-robot collaboration in a shared workplace. Robot Comput Integr Manuf 56:233-243.

[183] Nikolakis N, Sipsas K, Tsarouchi P, Makris S (2018) On a shared human-robot task scheduling and online re-scheduling. Procedia CIRP 78:237-242.

[184] Nonaka Y, Erdős G, Kis T, Nakano T, Váncza J (2012) Scheduling with alternative routings in CNC workshops. CIRP Ann - Manuf Technol 61(1):449454.

[185] Ong S-K, Chong JWS, Nee AYC (2006) Methodologies for immersive robot programming in an augmented reality environment. Proc. 4th Int. Conf Comput. Graph. Interact. Tech. Australas. Southeast Asia. ACM, pp 237-244.

[186] Orlandini A, Finzi A, Cesta A, Fratini S Generating Controllers for Flexible Plan Execution: a TGA approach. ICAPS Work. Valid. Verif. Plan. Sched. Syst.

[187] Osada T, Yano K (2009) Collision avoidance control with dynamical potential method for meal-assist robot. IFAC Proc. IFAC, pp 585-590.

[188] Oyekan J0, Hutabarat W, Tiwari A, Grech R, Aung MH, Mariani MP, LópezDávalos L, Ricaud T, Singh S, Dupuis C (2019) The effectiveness of virtual environments in developing collaborative strategies between industrial robots and humans. Robot Comput Integr Manuf 55:41-54.

[189] Pandey AK, Alami R (2010) Mightability maps: A perceptual level decisional framework for co-operative and competitive human-robot interaction. Intell. Robot. Syst. (IROS), 2010 IEEE/RSJ Int. Conf. IEEE, pp 5842-5848.

[190] Park J-J, Lee Y-J, Song J-B, Kim H-S (2008) Safe joint mechanism based on nonlinear stiffness for safe human-robot collision. Robot. Autom. 2008. ICRA 2008. IEEE Int. Conf. IEEE, pp 2177-2182.

[191] Park JJ, Hyun WJ, Mun SC, Park YT, Park OO (2015) Highly stretchable and wearable graphene strain sensors with controllable sensitivity for human motion monitoring. ACS Appl Mater Interfaces 7(11):6317-6324.

[192] Pavlovic VI, Sharma R, Huang TS (1997) Visual interpretation of hand gestures for human-computer interaction: A review. IEEE Trans Pattern Anal Mach Intell 19(7):677-695.

[193] Pearce M, Mutlu B, Shah J, Radwin R (2018) Optimizing makespan and ergonomics in integrating collaborative robots into manufacturing processes. IEEE Trans Autom Sci Eng (99):1-13.

[194] Pellegrinelli S, Admoni H, Javdani S, Srinivasa S (2016) Human-robot shared workspace collaboration via hindsight optimization. Intell. Robot. Syst. (IROS), 2016 IEEE/RSJ Int. Conf. IEEE, pp 831-838.

[195] Pellegrinelli S, Moro FL, Pedrocchi N, Tosatti LM, Tolio T (2016) A probabilistic approach to workspace sharing for human-robot cooperation in assembly tasks. CIRP Ann - Manuf Technol 65(1):57-60.

[196] Pellegrinelli S, Orlandini A, Pedrocchi N, Umbrico A, Tolio T (2017) Motion planning and scheduling for human and industrial-robot collaboration. CIRP Ann - Manuf Technol 66(1):1-4.

[197] Pérez L, Rodríguez Í, Rodríguez N, Usamentiaga R, García DF (2016) Robot guidance using machine vision techniques in industrial environments: A comparative review. Sensors 16(3):335.

[198] Petruck H, Faber M, Giese H, Geibel M, Mostert S, Usai M, Mertens A, Brandl C (2019) Human-Robot Collaboration in Manual Assembly - A Collaborative Workplace. Adv Intell Syst Comput 825:21-28.

[199] Pfitzner C, Antal W, Hess P, May S, Merkl C, Koch P, Koch R, Wagner M (2014) $3 \mathrm{~d}$ multi-sensor data fusion for object localization in industrial applications. ISR/Robotik 2014; 41st Int. Symp. Robot. Proc. VDE, pp 1-6.

[200] Pilz GmbH \& Co. KG (2016) Safetyeye.

[201] Pintzos G, Rentzos L, Papakostas N, Chryssolouris G (2014) A novel approach for the combined use of AR goggles and mobile devices as communication tools on the shopfloor. Procedia CIRP 25:132-137.

[202] Pohlt C, Haubner F, Lang J, Rochholz S, Schlegl T, Wachsmuth S (2019) Effects on User Experience during Human-Robot Collaboration in Industria Scenarios. Proc. - 2018 IEEE Int. Conf. Syst. Man, Cybern. SMC 2018. , pp 837842.

[203] Prassler E, Bruyninckx H, Nilsson K, Shakhimardanov A (2009) The use of reuse for designing and manufacturing robots - White Paper on the reuse of hardware and software components and architectural patterns in the design of robot systems and applications.

[204] Py F, Rajan K, McGann C (2010) A systematic agent framework for situated autonomous systems. Proc. 9th Int. Conf. Auton. Agents Multiagent Syst. Vol. 2Volume 2. International Foundation for Autonomous Agents and Multiagent Systems, pp 583-590. 
[205] Qiao G, Weiss BA (2017) Accuracy degradation analysis for industrial robot systems. ASME 2017 12th Int. Manuf. Sci. Eng. Conf. collocated with JSME/ASME 2017 6th Int. Conf. Mater. Process. American Society of Mechanical Engineers, p V003T04A006-V003T04A006.

[206] Quigley M, Conley K, Gerkey B, Faust J, Foote T, Leibs J, Wheeler R, Ng AY (2009) ROS: an open-source Robot Operating System. ICRA Work. open source Softw. Kobe, Japan, p 5.

[207] Rajnathsing H, Li C (2018) A neural network based monitoring system for safety in shared work-space human-robot collaboration. Ind Robot An Int J 45(4):481-491.

[208] Ranza F, Komendaa T, Reisingera G, Holda P, Hummelc V, Sihna W (2018) A Morphology of Human Robot Collaboration Systems for Industrial Assembly. Procedia CIRP 72(1):99-104.

[209] Reinhart G, Munzert U, Vogl W (2008) A programming system for robot-based remote-laser-welding with conventional optics. CIRP Ann - Manuf Technol 57(1):37-40.

[210] Rentzos L, Papanastasiou S, Papakostas N, Chryssolouris G (2013) Augmented reality for human-based assembly: using product and process semantics. IFAC Proc Vol 46(15):98-101.

[211] Rogalla 0, Ehrenmann M, Zollner R, Becher R, Dillmann R (2002) Using gesture and speech control for commanding a robot assistant. Robot Hum. Interact Commun. 2002. Proceedings. 11th IEEE Int. Work. IEEE, pp 454-459.

[212] Rosenstrauch MJ, Krüger J (2018) Safe human robot collaboration-Operation area segmentation for dynamic adjustable distance monitoring. 2018 4th Int Conf. Control. Autom. Robot. IEEE, pp 17-21.

[213] Rybski P, Anderson-Sprecher P, Huber D, Niessl C, Simmons R (2012) Sensor fusion for human safety in industrial workcells. IEEE Int. Conf. Intell. Robot. Syst. , pp 3612-3619.

[214] Sadrfaridpour B, Wang Y (2018) Collaborative assembly in hybrid manufacturing cells: An integrated framework for human-robot interaction. IEEE Trans Autom Sci Eng 15(3):1178-1192.

[215] De Santis A, Siciliano B, De Luca A, Bicchi A (2008) An atlas of physical humanrobot interaction. Mech Mach Theory 43(3):253-270.

[216] Schiavi R, Bicchi A, Flacco F (2009) Integration of active and passive compliance control for safe human-robot coexistence. Proc. - IEEE Int. Conf. Robot. Autom. , pp 259-264.

[217] Schlegl T, Kröger T, Gaschler A, Khatib O, Zangl H (2013) Virtual whiskersHighly responsive robot collision avoidance. Intell. Robot. Syst. (IROS), 2013 Highly responsive robot collision avoidanc
IEEE/RSJ Int. Conf. IEEE, pp 5373-5379.

[218] Schmidt B, Wang L (2014) Depth camera based collision avoidance via active robot control. J Manuf Syst 33(4):711-7118.

[219] Schmidtler J, Bengler K (2018) Influence of Size-Weight Illusion on Usability in Haptic Human-Robot Collaboration. IEEE Trans Haptics 11(1):85-96.

[220] Schmidtler J, Knott V, Hölzel C, Bengler K (2015) Human Centered Assistance Applications for the Working Environment of the Future. Occup Ergon 12(3):83-95

[221] Shauri RLA, Saiki K, Toritani S, Nonami K (2012) Sensor Integration and Fusion for Autonomous Screwing Task by Dual-Manipulator Hand Robot. Procedia Eng 41:1412-1420.

[222] Shiller Z (2015) Off-line and on-line trajectory planning. Motion Oper. Plan. Robot. Syst. Springer, pp 29-62.

[223] Siciliano B, Khatib 0 (2008) Springer Handbook of Robotics. Springer Science \& Business Media

[224] Siemens (2019) Siemens Tecnomatix. In: Siemens. https://www.plm.automation.siemens.com/global/en/products/tecnomatix/.

[225] Surdilovic D, Radojicic J (2007) Robust control of interaction with haptic interfaces. Robot. Autom. 2007 IEEE Int. Conf. IEEE, pp 3237-3244.

[226] Surdilovic D, Yakut Y, Nguyen TM, Pham XB, Vick A, Martin-Martin R (2010) Compliance control with dual-arm humanoid robots: Design, planning and programming. Humanoid Robot. (Humanoids), 2010 10th IEEE-RAS Int. Conf. IEEE, pp 275-281.

[227] Takata S, Hirano T (2011) Human and robot allocation method for hybrid assembly systems. CIRP Ann - Manuf Technol 60(1):9-12.

[228] Tan JTC, Arai T (2011) Triple stereo vision system for safety monitoring of human-robot collaboration in cellular manufacturing. Proc - 2011 IEEE Int Symp Assem Manuf ISAM 2011. doi: 10.1109/ISAM.2011.5942335

[229] Tellaeche A, Kildal J, Maurtua I (2018) A flexible system for gesture based human-robot interaction. Procedia CIRP 72:57-62.

[230] Toivonen V, Ikkala K, Niemi E, Korhonen A (2006) Planning and scheduling system for make-to-order production. CIRP Ann - Manuf Technol 55(1):493496.

[231] Tonietti G, Schiavi R, Bicchi A (2005) Design and control of a variable stiffness actuator for safe and fast physical human/robot interaction. Robot. Autom. 2005. ICRA 2005. Proc. 2005 IEEE Int. Conf. IEEE, pp 526-531.

[232] Tsarouchi P, Athanasatos A, Makris S, Chatzigeorgiou X, Chryssolouris G (2016) High level robot programming using body and hand gestures. Procedia CIRP 55:1-5.

[233] Tsarouchi P, Makris S, Chryssolouris G (2016) On a human and dual-arm robot task planning method. Procedia CIRP 57:551-555.

[234] Tsarouchi P, Makris S, Chryssolouris G (2016) Human-robot interaction review and challenges on task planning and programming. Int J Comput Integr Manuf 29(8):916-931.

[235] Tsarouchi P, Makris S, Michalos G, Matthaiakis A-S, Chatzigeorgiou X Athanasatos A, Stefos M, Aivaliotis P, Chryssolouris G (2015) ROS based coordination of human robot cooperative assembly tasks-An industrial case study. Procedia CIRP 37:254-259.

[236] Unhelkar V V, Lasota PA, Tyroller Q, Buhai R-D, Marceau L, Deml B, Shah JA (2018) Human-Aware Robotic Assistant for Collaborative Assembly: Integrating Human Motion Prediction with Planning in Time. IEEE Robot Autom Lett 3(3):2394-2401.

[237] Váncza J, Monostori L, Lutters D, Kumara SR, Tseng M, Valckenaers P, Van Brussel H (2011) Cooperative and responsive manufacturing enterprises. CIRP Ann - Manuf Technol 60(2):797-820.

[238] Vanthienen D, Klotzbuucher M, De Schutter J, De Laet T, Bruyninckx H (2013) Rapid application development of constrained-based task modelling and execution using domain specific languages. Intell. Robot. Syst. (IROS), 2013 IEEE/RSJ Int. Conf. IEEE, pp 1860-1866.

[239] Vasic M, Billard A (2013) Safety issues in human-robot interactions. Robot. Autom. (ICRA), 2013 IEEE Int. Conf. IEEE, pp 197-204.

[240] Vidal T (1999) Handling contingency in temporal constraint networks: from consistency to controllabilities. J Exp Theor Artif Intell 11(1):23-45.

[241] Vogel C, Walter C, Elkmann N (2013) A projection-based sensor system for safe physical human-robot collaboration. IEEE Int. Conf. Intell. Robot. Syst. , pp 5359-5364.

[242] Vogel T, Almada-Lobo B, Almeder C (2017) Integrated versus hierarchical approach to aggregate production planning and master production scheduling. OR Spectr 39(1):193-229.

[243] Vogl W (2009) Eine interaktive räumliche Benutzerschnittstelle für die Programmierung von Industrierobotern. Herbert Utz Verlag

[244] Wang CCL, Chu C-H, Wang L, Ramani K (2014) Depth cameras based techniques and applications in design, manufacturing and services. J Manuf Syst 33(4):675-676

[245] Wang L (2015) Collaborative robot monitoring and control for enhanced sustainability. Int J Adv Manuf Technol 81(9-12):1433-1445.

[246] Wang L, Schmidt B, Givehchi M, Adamson G (2015) Robotic assembly planning and control with enhanced adaptability through function blocks. Int J Adv Manuf Technol 77(1-4):705-715.

[247] Wang L (2013) Machine availability monitoring and machining process planning towards Cloud manufacturing. CIRP J Manuf Sci Technol 6(4):263273.

[248] Wang L (2008) Wise-ShopFloor: an integrated approach for web-based collaborative manufacturing. Syst Man, Cybern Part C Appl Rev IEEE Trans 38(4):562-573

[249] Wang L (2015) An overview of function block enabled adaptive process planning for machining. J Manuf Syst 35:10-25.

[250] Wang L, Mohammed A, Onori M (2014) Remote robotic assembly guided by 3D models linking to a real robot. CIRP Ann - Manuf Technol 63(1):1-4.

[251] Wang L, Schmidt B, Nee AYC (2013) Vision-guided active collision avoidance for human-robot collaborations. Manuf Lett 1(1):5-8.

[252] Wang L, Wang XV (2018) Safety in Human-Robot Collaborative Assembly. Cloud-Based Cyber-Physical Syst. Manuf. Springer, pp 211-241.

[253] Wang L, Wang XV (2018) Cloud Robotics Towards a CPS Assembly System. Cloud-Based Cyber-Physical Syst. Manuf. Springer, pp 243-259.

[254] Wang P, Liu H, Wang L, Gao RX (2018) Deep learning-based human motion recognition for predictive context-aware human-robot collaboration. CIRP Ann - Manuf Technol 67(1):17-20.

[255] Wang S, Song J, Lien J, Poupyrev I, Hilliges O (2016) Interacting with soli: Exploring fine-grained dynamic gesture recognition in the radio-frequency spectrum. Proc. 29th Annu. Symp. User Interface Softw. Technol. ACM, pp 851860.

[256] Wang XV, Kemény Z, Váncza J, Wang L (2017) Human-robot collaborative assembly in cyber-physical production: Classification framework and implementation. CIRP Ann - Manuf Technol 66(4):5-8.

[257] Wang XV, Seira A, Wang L (2018) Classification, personalised safety framework and strategy for human-robot collaboration. CIE48 Proc. , pp 1-10.

[258] Wang XV, Wang L, Mohammed A, Givehchi M (2016) Ubiquitous manufacturing system based on Cloud: A robotics application. Robot Comput Integr Manuf 45:116-125.

[259] Weitschat R, Ehrensperger J, Maier M, Aschemann H (2018) Safe and efficient human-robot collaboration part I: Estimation of human arm motions. 2018 IEEE Int. Conf. Robot. Autom. IEEE, pp 1993-1999.

[260] Yamada Y, Hirasawa Y, Huang SY, Umetani Y (1996) Fail-safe human/robot contact in the safety space. Robot Hum. Commun. 1996., 5th IEEE Int. Work. IEEE, pp 59-64.

[261] Yanco HA, Drury JL (2004) Classifying human-robot interaction: an updated taxonomy. IEEE Int. Conf. Syst. Man Cybern. IEEE, pp 2841-2846.

[262] Yao B, Zhou Z, Wang L, Xu W, Liu Q, Liu A (2018) Sensorless and adaptive admittance control of industrial robot in physical human- robot interaction. Robot Comput Integr Manuf 51:158-168.

[263] Yao B, Zhou Z, Wang L, Xu W, Yan J, Liu Q (2018) A function block based cyberphysical production system for physical human-robot interaction. J Manuf Syst 48:12-23.

[264] Zhang D, Wang L, Esmailzadeh E (2006) PKM capabilities and applications exploration in a collaborative virtual environment. Robot Comput Integr Manuf 22(4):384-395.

[265] Zhao X, Pan J (2018) Considering Human Behavior in Motion Planning for Smooth Human-Robot Collaboration in Close Proximity. 2018 27th IEEE Int. Symp. Robot Hum. Interact. Commun. IEEE, pp 985-990. 